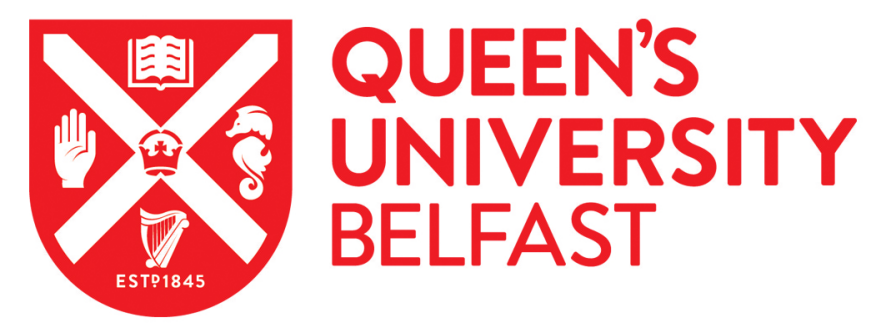

\title{
A Discussion on the Merits and Limitations of Using Drive-by Monitoring to Detect Localised Damage in a Bridge
}

Hester, D., \& González, A. (2017). A Discussion on the Merits and Limitations of Using Drive-by Monitoring to Detect Localised Damage in a Bridge. Mechanical Systems and Signal Processing, 90, 234-253.

https://doi.org/10.1016/j.ymssp.2016.12.012

Published in:

Mechanical Systems and Signal Processing

Document Version:

Peer reviewed version

Queen's University Belfast - Research Portal:

Link to publication record in Queen's University Belfast Research Portal

Publisher rights

(c) 2016 Elsevier Ltd. This manuscript version is made available under the CC-BY-NC-ND 4.0 license http://creativecommons.org/licenses/bync-nd/4.0/ which permits distribution and reproduction for non-commercial purposes, provided the author and source are cited.

\section{General rights}

Copyright for the publications made accessible via the Queen's University Belfast Research Portal is retained by the author(s) and / or other copyright owners and it is a condition of accessing these publications that users recognise and abide by the legal requirements associated with these rights.

Take down policy

The Research Portal is Queen's institutional repository that provides access to Queen's research output. Every effort has been made to ensure that content in the Research Portal does not infringe any person's rights, or applicable UK laws. If you discover content in the Research Portal that you believe breaches copyright or violates any law, please contact openaccess@qub.ac.uk. 
A Discussion on the Merits and Limitations of Using Drive-by Monitoring to Detect

\title{
Localised Damage in a Bridge
}

\author{
David Hester ${ }^{\text {a1 }}$, Arturo González ${ }^{\mathrm{b}}$
}

\author{
aSPACE, David Keir Building, Queen’s University Belfast, BT9 5AG, UK.
}

bSchool of Civil Engineering, University College Dublin, Belfield, Dublin 4, Ireland

Keywords: Bridges; Moving Load; Damage Detection; SHM; Wavelets.

Abstract. Given the large number of bridges that currently have no instrumentation, there are obvious advantages in monitoring the condition of a bridge by analysing the response of a vehicle crossing it. As a result, the last two decades have seen a rise in the research attempting to solve the problem of identifying damage in a bridge from vehicle measurements. This paper examines the theoretical feasibility and practical limitations of a drive-by system in identifying damage associated to localized stiffness losses. First, the nature of the damage feature that is sought within the vehicle response needs to be characterized. For this purpose, the total vehicle response is considered to be made of 'static' and 'dynamic' components, and where the bridge has experienced a localized loss in stiffness, an additional 'damage' component. Understanding the nature of this 'damage' component is crucial to have an informed discussion on how damage can be identified and localised. Leveraging this new understanding, the authors propose a wavelet-based drive-by algorithm. By comparing the effect of the 'damage' component to other key effects defining the measurements such as 'vehicle speed', the 'road profile' and 'noise' on a wavelet contour plot, it is possible to establish if there is a frequency range where drive-by can be successful. The algorithm uses then specific frequency bands to improve the sensitivity to damage with respect to limitations imposed by Vehicle-Bridge vibrations. Recommendations on the selection of the mother wavelet and frequency band are provided. Finally, the paper discusses the impact of noise and road profile

\footnotetext{
${ }^{1}$ Corresponding author, email address: d.hester@qub.ac.uk
} 
on the ability of the approach to identify damage and how periodic measurements can be effective at monitoring localised stiffness changes.

\subsection{Introduction}

With the increasing interest in drive-by monitoring to identify damage in a bridge, this paper presents a timely theoretical examination of the merits and potential limitations of the approach. Here, drive-by monitoring refers to recording vehicle acceleration signals as the vehicle passes over a bridge and then analysing these signals to identify damage in the bridge. To set the context of the study this introduction is broken in three sub-sections. Section 1.1 gives a brief overview of the area of bridge Structural Health Monitoring (SHM), section 1.2 looks specifically at the area of drive-by inspections and finally section 1.3 describes the objectives of this paper.

\subsection{Bridge SHM}

Mufti et al. [1] argue that SHM can reduce the cost of maintenance of existing bridges by providing owners with information that will enable them to carry out the most effective repair. Vibrationbased condition monitoring techniques, typically used in SHM to discern information about the bridge, have been discussed in several reviews [2, 3]. Essentially these approaches work by tracking changes in the modal parameters of the structure (e.g. frequencies and mode shapes). The latter are determined by the physical properties of the structures such as stiffness and mass, therefore any change in the physical properties (e.g., a localised or global loss of stiffness) will cause detectable changes in the modal properties [4].

Damage be can be identified by tracking changes in the natural frequency $[5,6]$. However, Salawu [7] points out that while resonant frequencies have the advantage of being easy to measure, the disadvantage is that changes in frequency can be due to environmental conditions as well as damage. Damage can also be identified by analysing the mode shape directly $[8,9]$ or by tracking changes in the mode shapes of the structure [10,11]. Broadly speaking mode shapes are 
significantly less affected by environmental effects than natural frequencies. However, a disadvantage of mode shape based approaches lies in the requirement of many sensors for an accurate definition of their shape. Other authors have identified damage in bridge/beam structures using Finite Element Model (FEM) updating [12, 13]. While FEM updating techniques have been shown to provide high levels of accuracy, they rely on accurate and sufficient experimental data for model calibration which sometimes can prove to be difficult or expensive to undertake. Finally, a number of authors have placed emphasis upon identifying localised damage in a beam from its response to a moving force. For example, while Zhu \& Law [14] and Roveri \& Carcaterra [15] apply wavelet analysis and Hilbert-Huang transform respectively to the mid-span displacement response, Hester \& Gonzalez [16] apply wavelet analysis to the acceleration response, for identifying the location of damage in a beam.

The aforementioned methods are based on analysis of measurements by sensors installed on the structure. The difficulty/cost of installing instrumentation (on a signification proportion of the bridge stock) has led to increased research on drive-by inspection systems. Algorithms using indirect (vehicle) measurements are based on similar principles as those designed for direct measurements taken on the bridge. In addition to noise, difficulties associated with monitoring structures using drive-by systems are mainly twofold: (1) the sensitivity to damage decreases as the measurement location (i.e., the moving load) moves away from the damaged location, and (2) the separation of the content related to the bridge from content purely due to vehicle dynamics or road profile can be troublesome.

\subsection{Drive-by Inspections}

The increasing amount of research on the use of vehicle vibrations to discern information about the bridge is motivated by the large number of bridges that are not instrumented, and therefore the need for some alternative cost-efficient monitoring method [17]. Previous investigations have focused on the extraction of bridge frequencies from vehicle measurements [17]. The vehicle operates as an 
exciter of bridge frequencies and the vehicle also receives the bridge vibrations. Theoretical investigations show that, when tracked over a long period, this information could act as a useful reference for determining the degradation in stiffness or strength in the structure [18]. Experimental work by the same research group using a cart fitted with accelerometers towed behind a light commercial truck corroborates the findings of their earlier theoretical study [19]. A follow up theoretical study by the same authors examines the relative influence of the various dynamic parameters of the system on correctly identifying the bridge frequencies of concern [20], and a subsequent study looked at the effectiveness of different filtering methods to identify bridge fequencies [21]. Other authors have validated their drive-by algorithms via scaled laboratory experiments [22-26]. It must be noted that the level of resolution of the spectrum is related to the time that the vehicle is on the bridge, i.e., frequencies cannot be predicted accurately at high traffic speeds, and a-sufficient level of bridge excitation is needed [27]. Rather than using only changes in bridge frequency as a potential damage indicator, vehicle measurements are used by Kim et al [25], Kim et al-_[28], Gonzalez et al-_[29] and Keenahan et al-_[30] to track changes in structural damping and by Yang et al-[31] and Oshima et al- [32] to extract mode shapes, which can be subsequently employed for damage detection similarly to bridge SHM. In addition to dynamic characteristics of the bridge, McGetrick et al- [26] and OBrien et al- [33] demonstrate that a static mechanical property such as global stiffness can also be obtained from the vehicle response and used for damage detection purposes. - also track changes in the structural damping from vehicle measurements.

Using numerical simulations, Nguyen et al [34] are some of the first researchers to show that in principle drive-by systems can not only be used for identifying global damages (by tracking parameters such as frequencies or damping), but also localised damage. In particular, they analyse the body displacement experienced by a 2-axle vehicle crossing a beam using wavelets and they observe that small peaks in the wavelet coefficients occur as each axle crosses the damaged section 
of the beam [34]. In more recent work, Zhang et al [35] use the acceleration response of a vehicle (as it crosses the beam) to identify the location of damage in the beam. The method they use to identify damage is based on using operational deflected shape curvature and they demonstrate the effectiveness of the approach using both numerical simulaitons and experimental testing. Both authors $[34,35]$ report deteoriation in their results as the speed of the vehicle increases. In spite of all advances in indirect bridge monitoring using passing vehicles, a recent review by Malekjafarian et al [36] concludes that further investigations on the impact of vehicle speeds, noise and rough road profiles are needed before drive-by systems can be successfully implemented in practise. The sections below represent a step forward towards addressing these issues.

\subsection{Aims and scope}

This paper carries out numerical simulations of a vehicle crossing a damaged finite element beam model to examine the merits and potential limitations of the drive-by approach to detect localised damage in the bridge. The simulated vehicle signals are subsequently analysed to see if damage can be successfully detected. For this purpose, Section 2 shows that there is a sound theoretical basis for the approach, i.e. there is a characteristic feature (of bridge damage) in the vehicle response signal when it passes over a bridge that has experienced a localised loss in stiffness. Section 3 shows that signal processing can be used to identify bridge damage feature in the axle acceleration signal when the vehicle axle is modelled as a point force. However, this paper also shows that extracting that feature using signal processing techniques can be challenging and even infeasible for some scenarios. Therefore, Section 3 also shows why damage cannot be detected at high vehicle speeds. Similarly, Section 4 shows how that the inclusion of road profile and noise has a very negative effect on the ability of the approach to identify damage.

In summary the research presented in the current paper expands on previous work in three principal aspects: 
i. The origin and nature of the damage feature as a result of a localised stiffness loss is characterized. It is demonstrated from first principles why the vehicle response contains a characteristic feature of bridge damage. The shapes of the feature associated to displacement, velocity and acceleration responses are illustrated and discussed. This feature is used for the reasoning of the limitations of a drive-by system in capturing localised damage.

ii. Informed by the findings of (i), a signal processing approach using wavelets is proposed to extract the damage feature from the axle acceleration signal. Specifically a 2D wavelet analysis approach is used which allows anomalies in the signal to be captured (i.e., including those associated to weakened sections).

iii. Finally the limitations of drive-by monitoring, particularly in relation to vehicle speed, road profile and measurement noise are discussed. It is explained why other authors [28, 29] have observed difficulty in detecting damage as the speed of the vehicle increases.

In other words, this paper aims to highlight those scenarios where drive-by monitoring is portentially fesiable and also to identify scenarios were the performance of a drive-by system is likely to be limited.

\subsection{Demonstrating why the vehicle response contains a characteristic feature of bridge}

\section{damage}

The purpose of this section of the paper is to demonstrate that if a vehicle crosses a bridge that has experienced a localised loss of stiffness, the axle response signals (i.e. displacement, velocity and acceleration signals) will contain a characteristic feature denoting bridge damage.

When a sprung vehicle is travelling on a general section of roadway, the vertical displacement of the axle (and its time domain derivatives, i.e. velocity and acceleration) is primarily governed by 
two factors: (i) undulations in the road profile and (ii) the inertial forces in the vehicle-axle system. On the other hand, when a sprung vehicle is crossing a bridge, the total displacement of the axle (at time ' $t$ ') can be thought of as being made up of three displacements due to: (i) road profile $\left(d_{r}(t)\right)$, (ii) inertial forces $\left(\mathrm{d}_{\mathrm{i}}(\mathrm{t})\right)$, and (iii) the displacement of the bridge immediately under the axle $\left(\mathrm{d}_{\mathrm{b}}(\mathrm{t})\right)$. From the point of view of bridge damage detection, any information about the condition of the bridge will be contained in the $\mathrm{d}_{\mathrm{b}}(\mathrm{t})$ part of the axle response signal. Therefore, before trying to identify bridge damage by analysing the response signal of a crossing axle it is important to understand how bridge damage manifests in the $\mathrm{d}_{\mathrm{b}}(\mathrm{t})$ part of the axle signal. To be able to focus exclusively on the $d_{b}(t)$ part of the signal, the response of a Finite Element Model (FEM) to a moving point force (rather than a sprung axle) is simulated. For visualization purposes, this can be thought of as the displacement experienced by the contact point of the force with the bridge while crossing it. In particular, section 2.1 describes the formulation of the model and in section 2.2, the model is used to show how damage in the bridge affects the displacement, velocity and acceleration experienced by a point force. The findings of section 2.2 are subsequently used to inform the signal processing carried out in section 4 to identify damage in the bridge by analysing the axle acceleration response of a sprung mass vehicle model.

\subsection{Description of Finite Element Model}

The response of the bridge immediately under a moving point force is theoretically simulated to demonstrate the existence of a characteristic feature (of bridge damage) in the $\mathrm{d}_{\mathrm{b}}(\mathrm{t})$ part of the axle signal. The bridge is modelled as a discretised simply supported finite element beam model, and the response of the beam to a moving load is solved using the matrix differential equation given in Eq. (1)[37].

$$
M a(t)+C v(t)+K y(t)=f(t)
$$

where $\mathbf{y}(\mathbf{t})$ is a vector containing the displacement and rotation of the degrees-of-freedom of the model, and $\mathbf{v ( t )}$ and $\mathbf{a ( t )}$ their velocities and accelerations respectively. $\mathbf{M}, \mathbf{C}$ and $\mathbf{K}$ are the 
consistent global mass, global damping and global stiffness matrices of the bridge. Damping is typically low in bridges and its effect on the forced response is neglected here. The bridge model is composed of $401 \mathrm{D}$ discretised beam elements that are assembled into the global matrixes. The elemental stiffness and mass matrices for 1D beam elements are well known and can be found in various text books (e.g. [38]). The Wilson-theta method is the numerical integration technique used to solve Eq. (1) [16] with a time step $\Delta$ t of 0.0005 seconds.

Localised damage is introduced in the FEM via a localised loss of stiffness in the vicinity of a simulated crack. The stiffness reduction method proposed by Sinha et al [12] is used in this paper to model the effect of a crack. This method assumes a gradual loss of bending stiffness that extends 1.5 times the depth of the beam at both sides of the crack (Fig. 1). Earlier work on damaged beams has looked at rectangular beams, where the ratio of crack height $(h)$ to beam depth $(d)$ (denoted as delta in this paper) has often been used to portray the severity of the damage, e.g. [14, 34]. Using delta as a measure of damage severity preserves consistency with previous published work and permits meaningful comparison of results. Delta values of 0.1 and 0.2 signify $73 \%$ and $51 \%$ respectively of the $2^{\text {nd }}$ moment of area of a healthy rectangular section. The same equivalency between delta and associated percentage of the healthy $2^{\text {nd }}$ moment of area value is preserved for the beam sections used in this paper. The response of the damaged beam to a moving load are found to concur with those published by [14, 39].

\section{[Insert Fig. 1]}

The bridge modelled in this paper has a span length of $40 \mathrm{~m}$ and a width of $15 \mathrm{~m}$. The crosssectional properties are based on 10 SY6 precast concrete beams spaced at $1.5 \mathrm{~m}$ centres with a 195 mm thick deck slab. This type of cross section is typical of that used in a modern highway bridge of the same dimensions and results in an inertia of $6.02 \mathrm{~m}^{4}$ and a cross-sectional area of $10 \mathrm{~m}^{2}$. A 
Young's modulus of $3.5 \times 10^{10} \mathrm{~N} \mathrm{~m}^{-2}$ is adopted which leads to a first natural frequency of $2.88 \mathrm{~Hz}$ for the healthy structure.

\subsection{Components of Bridge Response}

The axle acceleration signal of a sprung vehicle is influenced by both the vehicle and bridge responses. To understand the type of vibrations the bridge imparts to the axle, it is useful to examine the theoretical vibrations in increasing order of complexity, i.e., in this section those vibrations experienced by a simple point force crossing a damaged bridge are examined first (Or more specifically, the vibrations experienced by the point of the bridge deck immediately underneath the force are examined). The y-axis of Fig. 2(a) shows the displacement under a constant force of 5 tonnes as it crosses the bridge at $6 \mathrm{~ms}^{-1}$, i.e., it is a plot of the bridge displacement immediately under the point load for every timestep. The bridge has a crack at the 1/3 point of the span with delta equal to 0.3 . The $x$-axis in the figure shows the normalized position of the force on the bridge $(\mathrm{x}(\mathrm{t}) / \mathrm{L}$ equal to 0 and 1 when the force is at the start and end of the bridge respectively. Figs. 2(b) and 2(c) show the velocity and acceleration immediately under the force. These are obtained by differentiating the displacement shown in Fig. 2(a) once and twice respectively with respect to time.

To understand why there is a characteristic damage feature in the displacement response as a force crosses the damaged bridge, it is useful to break the total displacement response under the point load (Fig. 2(a)) into a number of components, namely 'H static', 'dynamic' and 'damaged' (see Fig. 2(d)). The corresponding components of the velocity and acceleration signals are shown in Figs. 2(e) and (f) respectively. The meaning of the components in Fig. 2(d) can be understood as follows. ' $\mathrm{H}$ static' is the response that would be observed under the point load if the bridge was healthy and the load was moved incrementally across the bridge and applied statically at each discreet location. 
'damage' is the difference between the static response of the damaged and healthy structures, and it is found by subtracting ' $\mathrm{H}$ static' from ' $\mathrm{D}$ static (where ' $\mathrm{D}$ static' is the response observed under the point load as it is moved incrementally and applied statically across the structure when the bridge is damaged). Finally 'dynamic' response is due to inertial forces and it is calculated by subtracting 'D static' from the total response. It is acknowledged, that here the 'dynamic' component has healthy and damaged contents, while the healthy and damaged contents of the static component have been separated into ' $\mathrm{H}$ static' and 'damage' sub-components respectively. Certainly, the frequencies associated to the 'dynamic' component will change as a result of a localized damage, however, large severity damage levels and ideal conditions (low speed for high resolution, smooth profile, clear gaps between vehicle and bridge frequencies, etc.) are necessary for damage to become clear in the spectrum recorded by a drive-by system. Therefore, the 'damage' component is used here as reference to evaluate the ability of the drive-by system to capture a localized damage in the time domain.

\section{[Insert Fig. 2]}

It should be noted that the 'damage' component of Fig. 2(d) is quite small compared to the ' $\mathrm{H}$ static' component and that the maximum value of the 'damage' component occurs when the force is at $0.33 \mathrm{~L}$, i.e., over the damaged section. If the ' $\mathrm{H}$ static', 'damage' and 'dynamic' components of displacement in Fig. 2(d) are added together, the result is the displacement signal shown in Fig. 2(a). Fig. 2(e) shows the three components of the velocity response, which are obtained by differentiating the relevant components of displacement with respect to time. Similarly, the three components of the acceleration signal (Fig. 2(f)) are established by differentiating the relevant components of the velocity signal. The sum of the three components in Figs. 2(e) and (f) is equal to the corresponding total response shown in Figs. 2(b) and (c) respectively. It can be seen that as the order of differentiation increases, the 'dynamic' component becomes progressively larger with respect to 'static' and 'damage' components which are more difficult to distinguish. In the case of 
acceleration, the ' $\mathrm{H}$ static' and 'damage' components are very small relative to the 'dynamic' component, thus, they practically look like straight lines in Fig. 2(f). However, a plot of Fig. 2(f) at the magnified scale of Fig. 3 shows that the 'H static' and 'damaged' components of acceleration are not straight lines. Once the load enters the bridge, the ' $\mathrm{H}$ static' component (or acceleration due to the static displacement of the point of contact of the load with the bridge) gradually increases until it reaches a maximum when the load is at mid-span, and then it gradually decreases as the load moves toward the support. In contrast, the 'damage' component shows a clear peak when the load is over the damage location. Essentially the 'damage' component is the characteristic damage feature to be sought in vehicle acceleration signals. This paper looks at how this 'damage' feature might be extracted from the vehicle acceleration signal using signal processing and the logistics of doing this are discussed in section 3 .

\section{[Insert Fig. 3]}

\subsection{Detecting bridge damage using wavelets when the vehicle is modelled as a point load}

The previous section has shown that the acceleration experienced by a point force crossing a bridge will contain a characteristic damage feature (i.e., a 'damage' component) if the bridge has experienced a localized loss in stiffness. Ultimately, the objective of this paper is to examine the feasibility of identifying this damage feature by analysing the axle acceleration signal of a crossing vehicle as reported in section 4. However, before moving on to axle acceleration signals (from a sprung vehicle model) it is informative to start with signals similar to those shown in Fig. 2, i.e. acceleration signals experienced by a point force crossing a bridge. These signals, referred here as 'point force' acceleration signals, and it is these kind of signals that are analysed in this section.

These 'point force' acceleration signals are, in effect, the acceleration under the point force while moving across the bridge. They are used as a simplified analogue of the axle acceleration of a real 
vehicle. However, it is important for the reader to understand that this is an approximation, i.e. the 'point force' acceleration is not the same thing as an axle acceleration signal. Here they are used to represent an ideal situation where the axle acceleration of a vehicle is affected only by its speed and by the deformation of the bridge due to the external applied force and the internal inertial forces of the bridge. I.e., this 'point force' model ignores the dynamic component of the axle force. It is acknowledged that, in reality, the force exerted by an axle is made of two components: a static component that remains constant and a dynamic component that varies as a result of interacting with the road irregularities and with the bridge. Therefore, this simplified model serves the purpose of highlighting limitations of drive-by monitoring, without the interference of vehicle dynamics that will tend to hinder any signs of bridge damage. In Section 4, a Vehicle-Bridge Interaction (VBI) simulation model is employed to generate more realistic acceleration signals that allow assessing the impact of the road profile and the dynamic characteristics of the vehicle on damage detection.

Even when dealing with point force acceleration signals (rather than the more complicated sprung axle accelerations), identifying damage can be a challenging task because 'as seen in Fig. 2(f)' the amplitude of the 'damage' component is very small relative to the 'dynamic' component and therefore the identification of damage in the point force acceleration signal (shown in Fig. 2(c)) is less than obvious. Therefore if the 'damage' feature is to be extracted from the point force acceleration response, some form of signal processing is required. To demonstrate the nuances of the challenge involved in extracting the damage feature, the authors needed to choose a signal processing technique. Ultimately, this paper uses wavelets for signal processing as they are effective at analysing local zones of a larger signal and they have already been successfully applied to similar SHM applications [14, 16, 34]. The authors are not necessarily arguing that wavelets are the best approach for this problem as in principle, other time-frequency methods could have been employed (e.g., Hilbert Huang transform). However, the type of limitations experienced by wavelet 
analysis (which are shown later in the paper) are equally relevant to other signal processing techniques that could be used. Section 3.1 shows how wavelet analysis can be applied to damage identification, section 3.2 provides a brief discussion on wavelet selection and finally, section 3.3 demonstrates why damage cannot be identified at some vehicle speeds.

\subsection{Using Wavelets to Identify Bridge Damage in Vehicle Accelerations.}

Wavelet analysis was developed to give better frequency-time information about a signal than traditional transforms such as the Fourier Transform. Hou et al [40] give some useful examples that illustrate the capacity of the wavelet transform to capture frequency-time information. A description of the most common wavelets and a rigorous mathematical definition of wavelet analysis is given in the literature [41-43], while a practical review of the uses of the wavelet transform in the field of SHM is given in [44].

In practical terms, a wavelet transform is implemented as follows: The wavelet of scale $s_{1}$ is compared to a section of the start of the time series signal $y(x)$, and a wavelet coefficient $\mathrm{Y}\left(\mathrm{x}_{1}, \mathrm{~s}_{1}\right)$ is calculated. $\mathrm{Y}\left(\mathrm{x}_{1}, \mathrm{~S}_{1}\right)_{\mathrm{s}}$ represents how similar the wavelet is to the start of the signal $\mathrm{y}(\mathrm{x})$, the greater the similarity the larger the value of $\mathrm{Y}\left(\mathrm{x}_{1}, \mathrm{~s}_{1}\right)$. Then the wavelet is moved to the next section of $\mathrm{y}(\mathrm{x})$ and the next wavelet coefficient $\mathrm{Y}\left(\mathrm{x}_{2}, \mathrm{~s}_{1}\right)$ is calculated. The entire length of the signal is checked in this way resulting in a plot of wavelet coefficients versus time. Subsequently the wavelet is scaled (i.e., stretched) and the process is repeated, resulting in wavelet coefficients $\mathrm{Y}\left(\mathrm{x}_{1}, \mathrm{~s}_{2}\right), \mathrm{Y}\left(\mathrm{x}_{2}, \mathrm{~s}_{2}\right)$, $\mathrm{Y}\left(\mathrm{x}_{3}, \mathrm{~s}_{2}\right)$, etc. Ultimately, the wavelet transform calculates a wavelet coefficient for each scale at each point in time. If the wavelet transform is applied to a continuous time series signal, the result is a 3-dimensional surface. If plotted in 3 dimensions, time and scale can be represented on two mutually perpendicular horizontal axes, and wavelet coefficient on the vertical axis. This 3D wavelet surface is commonly represented as a 2D contour plot. 
Fig. 4(a) shows the result of performing a wavelet transform on the acceleration signal of Fig. 2(c) using the Mexican hat wavelet (the reason for choosing the Mexican Hat wavelet is discussed in section 3.2). As before, the horizontal axis gives the normalised position of the load on the bridge $(x(t) / L)$. The vertical axis on the left shows the range of scales used in the wavelet transform. The magnitude of the absolute value of the wavelet coefficient $\left(\mathrm{Y}\left(\mathrm{x}_{\mathrm{i}}, \mathrm{S}_{\mathrm{j}}\right)\right)$ at a given scale, $\left(\mathrm{s}_{\mathrm{j}}\right)$, for a particular location of the load $\left(\mathrm{x}_{\mathrm{i}}\right)$ is indicated using colour, with a lighter colour indicating higher values of wavelet coefficient. The axis on the right shows the pseudo frequency corresponding to the scales shown on the axis on the left. Scale is related to pseudo frequency using Eq. (2),

where $F_{s}$ is the pseudo-frequency corresponding to scale $s$ in $\mathrm{Hz}, F_{c}$ is the centre frequency of the wavelet in $\mathrm{Hz}$ (For the Mexican Hat wavelet $\mathrm{F}_{\mathrm{c}}=0.25 \mathrm{~Hz}$ ), $\mathrm{s}$ is the scale of the wavelet, and $\Delta$ is the sampling period used to record the signal ( $\Delta=0.0005$ seconds in this simulation).

In Fig. 4(a), it is evident that at a scale of approximately 173 (corresponding to a pseudo-frequency of $2.88 \mathrm{~Hz}$ ), there is a series of high values for the wavelet coefficients. The latter is the $1^{\text {st }}$ natural frequency of the bridge. At scales of 500-1300, there are high wavelet coefficients at the edge of the plot, these being boundary effects due to the finite length of the input signal. In the same 500-1300 scale range, high values of wavelet coefficient are found when the load is at $x(t) / L=0.33$ (i.e., when the load crosses the damage). Fig. 4(b) shows the wavelet transform of the acceleration signal under a point load crossing a healthy bridge at $6 \mathrm{~ms}^{-1}$. This time, in the range of scales $500-1300$, apart from the boundary effects at the edge of the plot, there is an absence of high wavelet coefficients.

\section{[Insert Fig. 4]}


The increase in the values of wavelet coefficient as the load passes over the damaged section can be seen more clearly by examining a specific scale (note this is essentially taking a section through the contour plots shown in Fig. 4). Fig. 5 shows the wavelet coefficients at a scale of 700 for both the healthy and damaged bridges. The dashed plot in Fig. 5 is the result of analysing the acceleration signal shown in Fig. 2(c) with the Mexican hat wavelet at a scale of 700 . There is a clear peak at $x(t) / L=0.33$ that demonstrates the ability of the wavelet transform in detecting the 'damage' component of the acceleration signal. The solid plot in Fig. 5 is the result of analysing the acceleration response under the point load when it traverses the healthy bridge at a speed of $6 \mathrm{~ms}^{-1}$. This time there are no peaks in the plot, i.e. there is no 'damage' component in the acceleration signal.

\section{[Insert Fig. 5]}

Fig. 5 shows the results for a healthy bridge and a bridge that has a single damage (delta=0.3) at $x(t) / L=0.33$. Provided the damage locations are sufficiently far apart, it is possible to identify multiple damage locations. The solid black plot in Fig. 6 (delta=0.0) shows the results observed when the bridge has no damage, (i.e., this is a reproduction of the delta=0.0 plot from Fig. 5). The dashed plot in Fig. 6 shows the results of anaysing the 'point force' acceleration signal when the load crosses a bridge that has a delta $=0.2$ crack at $\mathrm{x}(\mathrm{t}) / \mathrm{L}=0.25$ and a delta $=0.1$ crack at $\mathrm{x}(\mathrm{t}) / \mathrm{L}=0.75$ (The wavelet scale used in the analysis is 700 and the speed of the point force is $6 \mathrm{~m} \mathrm{~s}^{-1}$ ). In the figure, it can be seen that the peaks in the dashed plot occur at the damage locations, namely $x(t) / L=0.25$ and $x(t) / L=0.75$. Moreover, the height of the peaks is proportionate to the severity of the damage, with the peak for the damage of delta=0.2 (@0.25L) being larger than peak corresponding to a damage of delta=0.1 (@0.75L). The heavy solid plot in Fig. 6 shows the results of anaysing the 'point force' acceleration signal when the load crosses a bridge that has a delta=0.1 crack at $x(t) / L=0.4$ and a delta $=0.2$ crack at $x(t) / L=0.6$. Again the peaks occur in the correct location and the height of the peaks is proportionate to the severity of damage. Simulations that had the 
damage locations even closer together were carried out, however, when the distance between the damages is less than approximately $0.2 \mathrm{~L}$, identifying the location of each individual damage became less reliable.

\section{[Insert Fig. 6]}

While the figures shown so far are somewhat simplified in the sense that the signals were produced using a simple moving constant load model, they are useful to demonstrate the fundamental principles behind detecting bridge damage by carrying out a wavelet analysis on the vehicle acceleration signal. The lessons learned at this preliminary stage will later be exploited when trying to detect damage in the more realistic situation of a vehicle interacting with the bridge while travelling over a rough profile.

\subsection{Wavelet selection}

The previous section shows that the Mexican Hat wavelet can be used to identify bridge damage when analyzing 'point force' acceleration signals. Essentially, there are two reasons for selecting Mexican Hat wavelet. Firstly, the Mexican hat wavelet has been used successfully by other authors $[14,16]$ to identify bridge damage when analyzing directly measured bridge acceleration response to a moving load, therefore applying them to the axle acceleration signal of a crossing vehicle seemed like a sensible approach. Secondly, the shape of the Mexican had wavelet (shown in Fig. 7(a)) is somewhat similar to the shape of the damage feature in Fig. 3, and it was felt that using an analysing wavelet similar in shape to the feature to be identified increased the probability of identify the damaged feature. Fig. 7(b) shows the Gauss 2 wavelet, which due to its relativity similar shape to the damage feature in Fig. 3, is also expected to be a suitable wavelet. For comparison purposes, Fig. 7(c) shows the Gauss 8 wavelet, which due to its shape it is considered unlikely to be as effective at identifying damage as the Mexican Hat or Gauss 2 wavelets. To illustrate this point, Fig. 8 shows the result of analyzing the point force acceleration signals shown 
in Fig. 2(c) with the three different wavelets shown in Fig. 7 (Note, the bridge used to generate the signal in Fig. 2(c) has a damage of delta=0.3 at $x(t) / L=0.33)$.

When discussing Fig. 4, it has been explained that damage needs to be captured in the range of scales above the bridge natural frequency. For the Mexican hat wavelet, it was shown in Fig. 4 that this zone existed between scales of 500-1300, which corresponded to pseudo frequencies of $1 \mathrm{~Hz}$ to $0.38 \mathrm{~Hz}$. To make a fair comparison between the three wavelets bases (Mexican Hat, Gauss 2 and Gauss 8). the scales used for the Gauss 2 and Gauss 8 wavelets (950 and 1300 respectively) correspond to pseudo frequencies in the range $1 \mathrm{~Hz}$ to $0.38 \mathrm{~Hz}$. The dashed plot in Fig. 8 shows the wavelet coefficients obtained when the signal is analysed with the Mexican Hat wavelet at a scale of 700 (essentially this is a reproduction of the dashed plot shown in Fig. 5). The solid plot in the figure shows the wavelet coefficients obtained when the signal is analysed with the Gauss 2 wavelet at a scale of 950. Similar to the Mexican hat wavelet, the Gauss 2 wavelet clearly identifies the damage. Overall the shape of the plot for Gauss 2 resembles the plot for Mexican Hat which is not surprising as both wavelets are very similar. Finally the heavy solid plot in Fig. 8 shows the result when the signal is analysed with the Gauss 8 wavelet at a scale of 1300 . Although the damage peak is still evident, this time it is less distinct than it was for Mexican hat and Gauss 2. The plots in Fig. 8 demonstrate that the closer the shape of the analyzing wavelet is to the shape of the damage feature, the better the chance of the wavelet identifying the damage. The analysis in Fig. 8 also shows that either Mexican Hat or Gauss 2 wavelets would be suitable for detecting bridge damage in axle acceleration signals, however for consistency with previous published work it was decided to move forward using Mexican Hat wavelet.

\section{[Insert Fig. 7]}

\section{[Insert Fig. 8]}




\subsection{Impact of Load Speed on the Range of Scales where Damage can be Detected}

The plots in Fig. 4 are for a load speed of $6 \mathrm{~ms}^{-1}$ and it can be seen that there is a broad range of scales (500-1300) that can be used to identify damage. However, it is important to understand that the frequency of the 'damage' component is affected by the speed of the load. Fig. 9(a) shows the theoretical 'damage' component of the acceleration signal for load speeds of 6,12 and $24 \mathrm{~ms}^{-1}$. It can be seen that the basic shape of the 'damage' component is the same for all speeds. However, as the speed of the load increases, the amplitude increases and more importantly the signal shortens in the time domain, leading to a higher frequency content. Figs. 9(b)-(d) show the wavelet transform of the 'damage' components shown in Fig. 9(a) (i.e. for load speeds of 6, 12 and $24 \mathrm{~ms}^{-1}$ ). It can be seen that the scales at which damage can be detected get progressively lower as the speed of the load increases. For a load speed of $24 \mathrm{~ms}^{-1}$, the wavelet coefficients of the 'damage' component occupy a range of scales $100-400$, which is within the range of scales occupied by the first natural frequency of the bridge (see Fig. 4). The significance of this is that, for sufficiently high load speeds, the 'damage' component will have the same frequency as the (much larger) 'dynamic' component (Fig. 2(f)). As a result, the wavelet transform will not be able to distinguish between the ‘damage’ and ‘dynamic’ components to identify damage.

\section{[Insert Fig. 9]}

\subsection{Detecting bridge damage using wavelets when vehicle is simulated using a 2-axle sprung model}

From a theoretical point of view, the analysis in sections 2 and 3 demonstrates the nature of the characteristic damage feature and shows how one might go about detecting it. It also demonstrates the practical limitations of a high vehicle speed (i.e., damage feature hindered by a main frequency of the bridge). However, it is important to remember that in the previous sections, the vehicle axle was modelled as a point force and it does not take into account that the vehicle axle is part of a larger vehicle system, including the influence of a rough road profile, vehicle dynamics, dynamic 
interaction with the bridge and noise. Section 4.1 describes the VBI model that will be used to address these issues. Section 4.2 demonstrates the difficulties experienced when trying to detect bridge damage by analysing acceleration signals that allow for vehicle oscillations excited by the movement of the bridge (i.e., the 'damage' feature needs to be isolated not only from the modes of vibration of the bridge as in Section 3, but also from modes of vibration of the vehicle). These difficulties get more serious in section 4.3 when a road profile is used to further excite the vehicle (i.e., road, vehicle and/or bridge frequencies corrupt most of the frequency range where the ‘damage’ feature can be captured). An approach is suggested to overcome some of these difficulties and its sensitivity to noise, which masks the 'damage' feature even further, is discussed in section 4.4.

\subsection{Description of Vehicle-Bridge Interaction (VBI) Model.}

The half-car vehicle model used in the simulations is shown in Fig. 10. It has 4 degrees of freedom, namely a vertical displacement for each of the two axles ( $\mathrm{y}_{1}$ and $\left.\mathrm{y}_{2}\right)$, as well as the bounce ( $\left.\mathrm{y}_{\mathrm{b}}\right)$ and pitch $\left(\varphi_{p}\right)$ of the body mass $[16,45]$. The body has moment of inertia $I_{p}$ for pitch and mass $m_{b}$ that is supported on two suspension assemblies (one per axle) that have stiffness $K_{s(1,2)}$ and damping

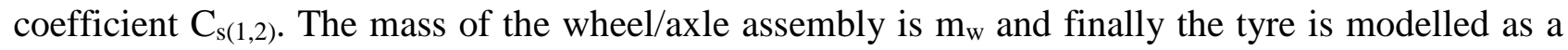
spring with stiffness $\mathrm{K}_{\mathrm{t}(1,2) \text {. }}$

\section{[Insert Fig. 10]}

Table 1 provides the parameters of the vehicle [46, 47]. The natural frequencies of the vehicle for bounce, pitch, and front and rear axle hops are $1.43 \mathrm{~Hz}, 2.07 \mathrm{~Hz}, 8.60 \mathrm{~Hz}$ and $10.22 \mathrm{~Hz}$ respectively. Pitching and rolling body frequencies are between $1.5 \mathrm{~Hz}$ and $4.5 \mathrm{~Hz}$ and hopping axle frequencies between $8 \mathrm{~Hz}$ to $16 \mathrm{~Hz}$, in agreement with typical values published by [48]. However, vehicle weights and stiffness may vary with respect to the selected values. If the changes in weight and stiffness of the vehicle were significant, they could affect the frequency of the vehicle-bridge 
system [49]. The bridge response will increase with vehicle weight, therefore, the relative increment in the static component of the bridge response will be more significant than the relative increment in dynamic component. The latter is corroborated by Cantero et al. [46] and González et al. [50] among others, who note that dynamic amplification factors associated to heavier vehicles are generally smaller than those associated to lighter vehicles. This paper has provided evidence on how the dynamic component will likely mask a localized damage, and hence, the lighter the vehicle, the more difficulties will be found in locating damage. In the case of a vehicle using high stiffness $\underline{\text { tires, the crossing over a localized bridge damage will be felt strongly leading to a more favorable }}$ scenario for damage detection than low stiffness tires. The advantages of high values of vehicle mass and stiffness in drive-by are highlighted by results from lab experiments reported by [24-26].

They test drive-by monitoring via scaled physical models with high vehicle to bridge mass ratios and high vehicle stiffness compared to full scale structures, and they achieve levels of accuracy that have not been demonstrated in the field yet.

Table 1. Parameters of vehicle model.

\begin{tabular}{|l|l|l|}
\hline Dimensional Data & & \\
\hline dimensions $(\mathrm{m})$ & wheel base $(\mathrm{D})$ & 5.5 \\
\hline & dist from centre of mass to front axle $\left(\mathrm{D}_{1}\right)$ & 3.66 \\
\hline & dist from centre of mass to rear axle $\left(\mathrm{D}_{2}\right)$ & 1.84 \\
\hline & & \\
\hline \multirow{2}{*}{ Mass and Inertia } & & \\
\hline \multirow{2}{*}{ mass $(\mathrm{kg})$} & front wheel/axle mass $\left(\mathrm{m}_{\mathrm{w} 1}\right)$ & 700 \\
\hline & rear wheel/axle mass $\left(\mathrm{m}_{\mathrm{w} 2}\right)$ & 1,100 \\
\hline & sprung body mass $\left(\mathrm{m}_{\mathrm{b}}\right)$ & 13,300 \\
\hline inertia $\left(\mathrm{kg} \mathrm{m}^{2}\right)$ & pitch moment of inertia of truck $\left(\mathrm{I}_{\mathrm{p}}\right)$ & 41,008 \\
\hline & & \\
\hline \multirow{2}{*}{ Suspension } & & \\
\hline \multirow{2}{*}{ spring stiffness $\left(\mathrm{kN} \mathrm{m}^{-1}\right)$} & front axle $\left(\mathrm{K}_{\mathrm{s} 1}\right)$ & 400 \\
\hline & rear axle $\left(\mathrm{K}_{\mathrm{s} 2}\right)$ & 1,000 \\
\hline damping $\left(\mathrm{kN} \mathrm{s} \mathrm{m}^{-1}\right)$ & front axle $\left(\mathrm{C}_{\mathrm{s} 1}\right)$ & 10 \\
\hline & rear axle $\left(\mathrm{C}_{\mathrm{s} 2}\right)$ & 10 \\
\hline tyre stiffness $\left(\mathrm{kN} \mathrm{m}^{-1}\right)$ & front axle $\left(\mathrm{K}_{\mathrm{t} 1}\right)$ & 1,750 \\
\hline & rear axle $\left(\mathrm{K}_{\mathrm{t} 2}\right)$ & 3,500 \\
\hline
\end{tabular}


Modelling the response of a VBI system is complex as there are two sub-systems (the moving vehicle and the bridge), that interact with each other via the contact forces that exist between the wheels and the bridge surface. Therefore, the problem is coupled and time dependant [51]. It is necessary to solve both subsystems while ensuring compatibility at the contact points (i.e., displacements of the bridge and the vehicle being the same at the contact point of the wheel with the roadway) [52]. In this paper, the response of each subsystem is solved independently using an iterative uncoupled procedure that ensures force equilibrium and geometric compatibility at each point in time [53-56].

\subsection{Testing damage detection in axle accelerations from VBI model with a smooth road profile.}

Fig. 11(a) shows the acceleration signal from axle 1 when the vehicle crosses the healthy bridge at a speed of $6 \mathrm{~ms}^{-1}$. The vertical dashed lines in the figure indicate when the axle 1 enters and leaves the bridge. The solid plot in Fig. 11(b) shows the result of analyzing the acceleration signal shown in part (a) with the Mexican hat wavelet at a scale of 700. The dashed plot in Fig. 11(b) shows the result of analyzing the axle acceleration signal when the vehicle crosses a damaged bridge (delta=0.3, @0.33L) at a speed of $6 \mathrm{~ms}^{-1}$. It is evident that unlike the case for a point load (Fig. 5), analyzing the axle acceleration signal from a half-car model at a single wavelet scale is not sufficient to identify damage.

\section{[Insert Fig. 11]}

However, Figures 4(a) and 6(b) showed that there are several scales between 700-1300 with high values of wavelet coefficients as the load crossed over the damage. Hester and Gonzalez [16] deal with a similar challenge when trying to identify damage from applying the wavelet transform directly to bridge accelerations. They propose to increase the sensitivity of the transformed 
accelerations to damage by splitting the range of scales, i.e., 700-1300 in this case into a series of strips as shown in Fig. 12 and by calculating an average value of wavelet coefficient for each strip $\left(\mathrm{W}_{\mathrm{s}}\right)$. Then, if one strip shows a dominant value of $\mathrm{W}_{\mathrm{s}}$, this indicates that there is damage in this zone. Rather than simply plotting $\mathrm{W}_{\mathrm{s}}$ with respect to the position of the strip, Hester and Gonzalez [16] find that plotting $\mathrm{W}_{\mathrm{n}}=\left(\mathrm{W}_{\mathrm{s}}^{2}-\mathrm{W}_{\mathrm{m}}^{2}\right)$ gives clearer results, where $\mathrm{W}_{\mathrm{m}}$ is the mean wavelet coefficient for all strips, and $\mathrm{W}_{\mathrm{n}}$ is here referred to as the normalised wavelet coefficient for the strip.

\section{[Insert Fig. 12]}

The solid plot in Fig. 13 (delta=0.0) shows the result of applying the approach of [16] to the (healthy) axle acceleration signal shown in Fig. 11(a) with strip widths of $0.5 \mathrm{~m}$. The heavy dashed plot_delta=0.3@0.33L) shows the result of analyzing the equivalent axle acceleration signal when there is delta $=0.3$ crack at the $1 / 3$ point of the span. The delta $=0.3$ plot in the figure shows a clear peak at $x(t) / L=0.33$, thereby indicating that there is damage at this location. On the other hand, the delta $=0.0$ (healthy) plot is everywhere close to zero, indicating that there is no localized loss of stiffness in the deck. The reason for plotting the $W_{n}$ signal only between $x(t) / L=0.25$ and $x(t) / L=0.8$ is to avoid the abrupt changes in wavelet coefficients as the axles enter and leave the bridge. Therefore, the normalized wavelet coefficients for the strips close to the supports are very high and mask any damage that might be present. As a result, damage cannot be detected close to the supports and only the portion of the plot between $x(t) / L=0.25$ and $x(t) / L=0.8$ gives meaningful results. The third plot in the figure (delta=0.2@0.33L \& delta=0.2@0.66L) shows the result of analyzing the axle acceleration signal when the bridge has a delta $=0.2$ crack at two locations, namely $0.33 \mathrm{~L}$ and $0.66 \mathrm{~L}$. It can be seen that there are distinct peaks at both damage locations.

\section{[Insert Fig. 13]}


The range of scales used to detect damage in Fig. 13 goes from 700 to 1300 . The lower scale limit was chosen so as to avoid interference from the main bridge frequency (see Fig. 4), i.e., at this scale the area of influence of the first natural frequency of the bridge has disappeared. The lower scale limit used is 4 times the scale corresponding to the $1^{\text {st }}$ natural frequency ( 4 x173 $\approx 700$ ). However, at very high scales, there is a loss of resolution and as a result, loss of accuracy in the location of the damage. For the $40 \mathrm{~m}$ bridge and a load speed of $6 \mathrm{~ms}^{-1}$, an upper scale limit of 1300 is found to be effective, i.e. an upper limit 7.51 times the scale corresponding to the first natural frequency $(7.51 \mathrm{x}$ $173=1300)$. The upper scale limit proposed here $(7.5 \mathrm{x}$ - first natural frequency) has in effect been identified empirically by trying a range of different upper limit scales from 900 to 1500 and a scale of 1300 (i.e., the scale corresponding to 7.5 times the first natural frequency) was found to give the best resolution for identifying the damage location. To examine if these scale ratios would prove effective for another bridge, they are applied to signals generated in the theoretical model of a $30 \mathrm{~m}$ bridge being traversed by a sprung vehicle moving at $6 \mathrm{~ms}^{-1}$.

The properties used to model the $30 \mathrm{~m}$ bridge are consistent with the properties of a concrete highway bridge spanning $30 \mathrm{~m}$, and the $1^{\text {st }}$ natural frequency is $4.1 \mathrm{~Hz}$. Axle acceleration signals are simulated in the same manner as for the $40 \mathrm{~m}$ bridge, i.e., the vehicle shown in Fig.10 moves across the bridge at $6 \mathrm{~ms}^{-1}$. Following a wavelet transform of the axle acceleration signal, the scale corresponding to the $1^{\text {st }}$ natural frequency of the $30 \mathrm{~m}$ bridge is found to be 121 . If the scale ratios used to detect damage in the $40 \mathrm{~m}$ bridge are applied to the $30 \mathrm{~m}$ span, lower and upper scale limits of 484 (4 x 121) and 909 (7.51 x 121) respectively are obtained. The result of applying these scale limits to the signal from the healthy bridge are shown as the solid plot (delta=0.0) in Fig. 14 and it can be seen that no dominant peaks are observed. When the same scale limits are applied to the axle acceleration signal when the bridge has a delta $=0.3$ crack at $x(t) / L=0.66$, the dashed plot in Fig. 14 results. This time there is a clear peak at $x(t) / L=0.66$, which shows that the lower and upper limit scale ratios observed on the $40 \mathrm{~m}$ bridge (for a vehicle speed of $6 \mathrm{~ms}^{-1}$ ) are also effective for the 30 
$\mathrm{m}$ bridge when the vehicle speed is $6 \mathrm{~ms}^{-1}$. However, it should be noted that the upper and lower limit scale ratios listed above can only be used as guidelines and need to be applied with caution. This is because the range of scales where the 'damage' component exists depends on the speed of the vehicle (see section 3.3). For sufficiently high speeds, the 'damage' component will co-exist in a narrow scale band together with the first bridge natural frequency and as a result there will be, in effect, no range of scales for which damage can be reliably identified.

\section{[Insert Fig. 14]}

\subsection{Testing damage detection algorithm on axle accelerations from VBI model with a rough road profile.}

The irregularities of the road profile have a big influence on the axle's acceleration signal and therefore these irregularities also affect the ability of a drive-by approach to identify damage. ISO 8608 (BS7853:1996) [57] classifies the roughness of a road profile into one of classes ' $A$ ' (excellent) to 'E' (very poor). A theoretical road profile of a given roughness class is generated according to Cebon [48] for use in numerical simulations. Fig. 15(b) shows the acceleration signal of axle 1 when the bridge has a crack of delta=0.3 at $0.33 \mathrm{~L}$ and the vehicle is travelling at $6 \mathrm{~ms}^{-1}$ on the class ' $A$ ' road profile shown in Fig. 15(a). If the acceleration signal in Fig. 11(b) (when the vehicle is travelling on a smooth profile), is compared to the acceleration signal in Fig. 15(b), two observations can be made: Firstly the inclusion of a road profile results in much larger amplitudes than when the vehicle was travelling on a smooth profile, and secondly the dominant frequency of vibration has increased. When the bridge has a smooth profile the dominant frequency of vibration in the axle was approximately $2.8 \mathrm{~Hz}$, i.e. the first natural frequency of the bridge. When a road profile is included the dominant frequency of vibration in the axle is approximately $8.6 \mathrm{~Hz}$, i.e. the 'hop’ frequency of axle 1. 
The normalized wavelet coefficient technique described in section 4.2 is applied to the acceleration

607 signal shown in Fig. 15(b) and the result is shown as the heavy dotted plot (delta=0.3) in Fig. 16. The solid plot (delta=0.0) in Fig. 16 is the result of analyzing the axle 1 acceleration signal when the bridge was healthy. Unlike the situation when the vehicle was travelling on a smooth profile (Fig. 13), this time there is no clear peak evident as the load passes over the damaged section. It can be seen that the delta $=0.0$ and delta=0.3 plots are basically indistinguishable from one another so the inclusion of a road profile has rendered damage undetectable using the current approach.

To understand why the inclusion of a road profile has such a detrimental effect on the ability of the technique to identify damage, it is useful to look back at Fig. 13. Although the plots in Fig. 13 are the result of analyzing axle acceleration signals, it is important to understand that the source of the peak in the delta=0.3 plot in Fig. 13 is actually the small extra displacement experienced by the axle as it crosses the damaged bridge, relative to what it experiences when it crosses the healthy bridge. From Fig. 2(d), it can be seen that in this case, this extra displacement is of the order of $0.1 \mathrm{~mm}$, however, the road profile in Fig. 15(a) results in vertical displacements of the order of $4 \mathrm{~mm}$. The displacement of axle 1 resulting from the irregularities of the road profile are a mixture of large amplitude low frequency displacements and low amplitude high frequency displacements. In the simulation under investigation, the axle acceleration signals obtained when the vehicle crosses a road profile are such that, when analysed at the range of scales where damage is known to occupy (i.e., 500-1300), the results for the healthy and damaged cases are practically the same. Essentially in the range of scales of interest to us (500-1300), the road profile appears to have a significantly greater impact than the characteristic damage feature. Consequently, it appears damage cannot be detected as the height of the road irregularities mask the extra bridge displacement caused by a localized stiffness loss. 
The road profile appears to govern the results of the wavelet analysis. This can be

demonstrated by calculating the acceleration response of a notional particle travelling over the 'solid' profile shown in Fig. 15(a) at $6 \mathrm{~ms}^{-1}$. 'Solid' in this instance signifies that no allowance is made for bridge deflection, and in this theoretical exercise no uplift of the particle is allowed. When the acceleration of this notional particle is analysed using the normalized wavelet coefficient technique, the dashed plot (particle) in Fig. 16 is obtained. This dashed plot is very similar to the healthy (delta $=0.0$ ) and damaged (delta $=0.3$ ) plots in the figure, which proves that the parts of the axle acceleration signal that correspond to the range of scales of interest (500-1300 in this case) are being dominated by vibrations resulting from the road profile rather than vibrations originating from the bridge and as a result, the characteristic feature for bridge damage remains hidden.

\section{[Insert Fig. 16]}

Therefore to identify damage it would be necessary to develop an approach that would allow us to separate the parts of the wavelet surface that are due to road profile from those that are due to damage. In principle, this could be achieved using an existing axle acceleration record (i.e., from a previous test) where the structure is presumed healthy. Admittedly, in practice, this could be difficult to implement, however, the aim of this study is to examine the merits and limitations of the approach so for the purposes of this paper it is assumed feasible as this allows us to examine other potential limitations. Having a previous acceleration signal from the presumed healthy bridge allows the calculation of a 'datum' wavelet surface. In all subsequent tests the axle acceleration is recorded and the 'current' wavelet surface is calculated. To remove the effect of road profile the 'datum' wavelet surface is simply subtracted from the 'current' wavelet surface. Here, the result of the subtraction is referred to as the 'residual' wavelet surface. By applying the technique described in section 4.2 to the residual wavelet surface a plot of the normalized wavelet coefficients (similar to those seen in Figs. 13 and 16) can be achieved and these are shown in Fig. 17 (Note that the results shown in Fig. 17 assume that a datum wavelet surface from the healthy bridge is available, 
i.e., delta=0.0). To generate Fig. 17, axle acceleration signals are simulated for the following bridge conditions: delta $=0.0$, delta $=0.1$, delta $=0.2$ and delta $=0.3$. For all damage severities, damage is assumed to occur at the $1 / 3$ point of the span. Fig. 17 shows the normalized wavelet coefficients calculated from the four residual wavelet surfaces corresponding to the four damage scenarios, (i.e. delta $=0.0-0.3$ ). When the structure remains undamaged (delta $=0.0$ ), the plot of the normalised wavelet coefficients shows no dominant peaks. Once damage occurs, a dominant peak starts to emerge at the damaged location, with the height of the peak increasing with the severity of the damage

\section{[Insert Fig. 17]}

\subsection{Testing damage detection in axle accelerations from VBI model with a rough road profile corrupted with noise.}

When assessing the suitability of a proposed damage detection method, its robustness with respect to noise needs to be considered. For this purpose, a noise vector is calculated using Eq. (3) in the procedure by Lyons [58].

$$
\text { noise }=\sqrt{\frac{\sigma^{2}}{\exp \left(\frac{S N R * \ln (10)}{10}\right)}} *[R]
$$

where $\sigma^{2}$ is the variance of the axle acceleration signal, SNR is the desired signal to noise ratio and [R] is a standard normal distribution vector with zero mean and unit standard deviation. The corrupted signal is simply the sum of the simulated theoretical acceleration signal and the noise vector calculated in Eq. (3). Then, the same approach used to generate Fig. 17 is applied to the corrupted acceleration signals, i.e., the 'datum' wavelet surface is subtracted from the 'current' wavelet surface to give a 'residual' wavelet surface. The normalized wavelet coefficients are then calculated from the 'residual' wavelet surface (Note that the acceleration signal used to calculate the ‘datum' wavelet surface contains noise as do all subsequent signals). SNRs of 70, 50 and 20 are 
used in the simulations and the results are presented in Figs. 18 (a), (b) and (c) respectively. For a SNR of 70, the plots are very similar to the noise free case (Fig. 17) in that when the structure remains undamaged (delta $=0.0$ ), the plot of the normalised wavelet coefficients shows no dominant peaks. Also once damage occurs, a dominant peak is observed at the $1 / 3$ point of the span, with the height of the peak increasing for more severe damage.

When the level of noise is increased to a SNR of 50, there are no dominant peaks for delta $=0.0$, so it can be established that there has been no loss of stiffness since the datum reading was taken. However, the delta=0.0 plot in part (b) of Fig. 18 is noticeably less smooth than the corresponding plot in part (a), and this is due to the increased noise in the acceleration signals. When small damage of delta $=0.1$ takes place, the plot in Fig. 18(b) does exhibit a small peak at the damage location, however the peak is not particularly dominant relative to the rest of peaks in the plot so the damage is not reliably identified. This shows that the presence of noise can mask small levels of damage and produce misleading peaks. For larger levels of damage (delta=0.2 and delta=0.3), the plots in Fig. 18(b) reveal clear peaks at the damage location which demonstrates that larger damage can still be detected for $\mathrm{SNR}=50$. In the case of $\mathrm{SNR}=20$, the noise has a detrimental effect on the ability of the approach to identify damage as not even delta $=0.3$ can be identified. The reasons for the poor performance are essentially two fold. Firstly, the 'damage' component is very small relative to the amplitude of the total acceleration signal and given that noise is added in proportion to the amplitude of the total acceleration signal, the noise shrouds the presence of damage. Secondly, the datum reading (which is included to overcome the impact of road profile) also contains noise, so in a sense any increase in noise has a doubly negative impact on the approach. In an effort to see if the impact of noise could be remediated by doing average results over multiple runs, 10 noisy signals were simulated, but the resulting plots were actually very similar to those shown in Fig. 18(c).

\section{[Insert Fig. 18]}




\subsection{Influence of Datum}

After establishing the limitations of the method with respect to noise, this section examines the sensitivity of the method to the selected datum. If the proposed method is applied to existing structures, it is possible that a subset of these structures will already have experienced a localized loss in stiffness, therefore the datum reading for his subset of structures will not correspond to a healthy state. However, it is found that even if the datum reading is taken on a bridge that is not in pristine condition, it will still allow a subsequent loss of stiffness to be identified. For example, a situation is assumed where a datum reading is taken in Year 1 on a bridge that has already experienced a delta=0.1 crack at the $1 / 3$ point of the span. Then, in Year 2 there is only minor deterioration as the severity of the crack increases to delta $=0.12$, but in Year 3 circumstances are such that there is a substantial further deterioration such that the severity of the crack reaches delta=0.22. Fig. 19 shows plots of the normalized wavelet coefficients for the assumed patterns of localized loss stiffness in Years 1 (delta=0.1), 2 (delta=0.12) and 3 (delta=0.22). In producing Fig. 19, the datum acceleration signal (Year 1) as well as all subsequent acceleration signals (Years 2 \& 3) have a SNR of 70. None of the peaks are dominant in the plot for Year 2, which indicates that there has been no significant stiffness degradation since the datum reading was taken. However, in Year 3, the peak at $\mathrm{x}(\mathrm{t}) / \mathrm{L}=0.33$ is dominant which indicates that there has been a substantial stiffness loss at this location since the since the datum reading was taken. This peak would flag a warning to the bridge manager for further investigations at this location to confirm the occurrence and exact extent of the stiffness loss.

\section{[Insert Fig. 19]}

\subsection{Conclusions}

The objective of this paper has been to present a theoretical examination of the merits and potential limitations of drive-by monitoring to identify localised stiffness loss in a bridge. It has been demonstrated that there is a theoretical basis for the axle response to contain a characteristic feature 
related to bridge damage. Understanding how this bridge damage feature manifests in the vehicle response is crucial, as it allows the practitioner develop bespoke algorithms to identify the bridge damage feature in measured axle acceleration signals. To be able to focus exclusively on the bridge contribution to the axle acceleration response (and thereby identify the bridge damage feature), a FEM of a point load crossing a bridge has been implemented. Using this model, it has been shown that the total acceleration experienced by a point load crossing a bridge can be divided into ' $\mathrm{H}$ static' (or static response that would be obtained if the structure was healthy), 'damage' (or difference between static response that would be obtained if the structures was damaged and ' $\mathrm{H}$ static') and 'dynamic' (the remainder of the total response).

Initially, using these simplified simulations (where the vehicle axle was modelled as a point force), it has been relatively easy for a simple signal processing technique to uncover this characteristic damage feature provided the speed of the load is low enough. This has also been proved for the case of multiple damaged locations. For higher speeds, it has been shown that the frequency of the 'damage' component will be too close to the first natural frequency of the bridge and damage will go unnoticed. Therefore, if the frequency content of the 'damage' component is close to the frequency of other vibrations in the Vehicle-Bridge system, it will become very difficult to distinguish damage for any signal processing technique.

When a VBI model has been employed, the problem of identifying bridge damage becomes more challenging because essentially the VBI model introduces more complex vibrations and more elaborate signal processing techniques are required. For example, when a vehicle is travelling on a smooth road profile, using a simple plot of wavelet coefficients versus time at a fixed scale as proposed in previous research, has appeared to be insufficient to detect damage. As a result, the authors have employed a more elaborate 2D wavelet analysis (wavelet coefficients versus scale and time) to seek for the damage in the frequency-time domain. During the investigation, it has been 
shown that those mother wavelets with a shape closer to the 'damage' component have provided best results. Damage has been extracted within a scale band (as opposed to a single scale) to reduce the negative impact associated with vibrations due to VBI. Using the more elaborate technique, bridge damage has been identified by a distinctive peak at the weakened portion of the bridge. This novel drive-by algorithm builds on previous work by the authors where they successfully apply the algorithm for detecting damage using direct bridge measurements [16]. Here, the authors have applied the algorithm to indirect vehicle accelerations by the first time.

When a more realistic situation of the vehicle travelling on a rough road profile was considered, detecting a localised stiffness loss by analysing just the current axle acceleration signal has become considerably more challenging and in some cases impractical. Fundamentally, the difficulty is that the source of the damage feature (in the axle acceleration signal) adds very small 'extra' displacement to the bridge due to the localised loss in stiffness, and when a road profile is included in the simulation this very small displacement is masked by the larger displacement of the axle displacement moving up and down on the road profile. Having identified the impracticality of identifying damage using just the current axle acceleration signal, the authors show that if a datum reading was available (e.g., a wavelet surface from last year when the bridge was assumed healthy), it is possible to identify damage. In particular, the drive-by algorithm has shown to successfully detect damage for a class ' $A$ ' profile and damage severity as low as delta=0.1 when a datum is used as reference. Here, it has been assumed that the road profile has not changed significantly. Otherwise, the datum needs to be re-calibrated. The approach has also proved to perform satisfactorily for signals corrupted with SNR of 70 (1.43\% noise). When SNR has been increased to 50 (2\% noise), it is not possible to distinguish delta=0.1, but delta $=0.2$ and higher are still noticeable with higher peaks associated to higher damage severities. These conclusions refer to specific bridge, vehicle, road and noise scenarios. For example, in the case of SNR of 20 (5\% noise), damage has not been identifiable. The proposed algorithm will be successful when the effect 
of the 'damage' component (i.e., variable with damage severity and location) is distinguishable from other effects derived from road profile and noise in the wavelet contour plot.

Although the use of a datum reading has proved to be effective, it is acknowledged that an accurate datum reading may not always be available for the road profile under investigation as a result of changes over time due to wear, rutting and occasional resurfacing works. Finally, even when an accurate datum reading was available, noise has been shown to have a very negative impact on the performance of the approach. For relatively low levels of noise (SNR=70 and 50), broadly speaking the approach has been able to detect damage, but with some expected deterioration in the results compared to the noise-free case. However, a SNR of 20 rendered the approach ineffective. In summary, there is a sound theoretical basis behind detecting and locating a localised loss in bridge stiffness by analysing an axle acceleration signal, however, there are a number of significant limitations to the approach, e.g. vehicle speed, noise and road roughness. Based on currently available technology, there are a number of substantial challenges to be overcome before drive-by can be reliably implemented in the field.

\section{References}

1. Mufti, A., et al., Are Civil Structural Engineers "Risk Averse”? Can Civionics Help? Sensing issues in civil structural health monitoring, 2005: p. 3-12.

2. Carden, E.P. and P. Fanning, Vibration based condition monitoring: a review. Structural Health Monitoring, 2004. 3(4): p. 355-377.

3. Fan, W. and P. Qiao, Vibration-based damage identification methods: a review and comparative study. Structural Health Monitoring, 2011. 10(1): p. 83-111.

4. Doebling, S.W., et al., Damage identification and health monitoring of structural and mechanical systems from changes in their vibration characteristics: a literature review, 1996, Los Alamos National Lab., NM (United States).

5. Messina, A., E. Williams, and T. Contursi, Structural damage detection by a sensitivity and statistical-based method. Journal of Sound and Vibration, 1998. 216(5): p. 791-808.

6. Nandwana, B.P. and S. Maiti, Detection of the location and size of a crack in stepped cantilever beams based on measurements of natural frequencies. Journal of Sound and Vibration, 1997. 203(3): p. 435-446.

7. Salawu, O., Detection of Structural Damage Through Change in Frequency: A review. Engineering Structures, 1997. 19: p. 718-723.

8. Hadjileontiadis, L.J., E. Douka, and A. Trochidis, Fractal dimension analysis for crack identification in beam structures. Mechanical Systems and Signal Processing, 2005. 19(3): p. 659-674. 
9. Pakrashi, V., A. O'Connor, and B. Basu, A study on the effects of damage models and wavelet bases for damage identification and calibration in beams. Computer-Aided Civil and Infrastructure Engineering, 2007. 22(8): p. 555-569.

10. Lee, J.J., et al., Neural networks-based damage detection for bridges considering errors in baseline finite element models. Journal of Sound and Vibration, 2005. 280(3-5): p. 555-578.

11. $\mathrm{Hu}, \mathrm{C}$. and M.T. Afzal, A statistical algorithm for comparing mode shapes of vibration testing before and after damage in timbers. Journal of Wood Science, 2006. 52(4): p. 348-352.

12. Sinha, J.K., M.I. Friswell, and S. Edwards, Simplified models for the location of cracks in beam structures using measured vibration data. Journal of Sound and Vibration, 2002. 251(1): p. 13-38.

13. Teughels, A. and G. De Roeck, Structural damage identification of the highway bridge Z24 by FE model updating. Journal of Sound and Vibration, 2004. 278(3): p. 589-610.

14. Zhu, X.Q. and S.S. Law, Wavelet-based crack identification of bridge beam from operational deflection time history. International Journal of Solids and Structures, 2006. 43(7): p. 22992317.

15. Roveri, N. and A. Carcaterra, Damage detection in structures under traveling loads by HilbertHuang transform. Mechanical Systems and Signal Processing, 2012. 28(0): p. 128-144.

16. Hester, D. and A. González, A wavelet-based damage detection algorithm based on bridge acceleration response to a vehicle. Mechanical Systems and Signal Processing, 2011. 28: p. 21.

17. González, A., P.J. McGetrick, and E.J. O'Brien. Detection of Bridge Dynamic Parameters Using an Instrumented Vehicle. in Proceedings of the Fifth World Conference on Structural Control and Monitoring, 5WCSCM. 2010. Tokyo, Japan.

18. Yang, Y.B., C.W. Lin, and J.D. Yau, Extracting bridge frequencies from the dynamic response of a passing vehicle. Journal of Sound and Vibration, 2004. 272(3-5): p. 471-493.

19. Lin, C.W. and Y.B. Yang, Use of a passing vehicle to scan the fundamental bridge frequencies: An experimental verification. Engineering Structures, 2005. 27(13): p. 1865-1878.

20. Yang, Y. and K. Chang, Extracting the bridge frequencies indirectly from a passing vehicle: Parametric study. Engineering Structures, 2009. 31(10): p. 2448-2459.

21. Yang, Y.B., K.C. Chang, and Y.C. Li, Filtering techniques for extracting bridge frequencies from a test vehicle moving over the bridge. Engineering Structures, 2013. 48(0): p. 353-362.

22. F. Cerda, J.G., J. Bielak, P. Rizzo, J. A. Barrera, Z. Zhang, S. Chen, M. McCann, and J. Kova. Indirect structural health monitoring in bridges: scale experiments. in Int. Conf. Bridge Maintenance, Safety and Management. 2012. Lago di Como, Italy.

23. Kim, C.W.K., M. \& Fujimoto T. Identifying bending stiffness change of a beam under a moving vehicle. in Fifth International Conference on Bridge Maintenance, Safety and Management, (IABMAS). 2012. Philadelphia, USA.

24. Cerda, F., et al., Indirect structural health monitoring of a simplified laboratory-scale model. Smart Structures and Systems, 2014. 13(4): p. 19.

25. Kim, C.W., et al., Drive-by bridge inspection from three different approaches. Smart Structures and Systems, 2014. 13(5): p. 21.

26. McGetrick, P.J., et al., Experimental validation of a drive-by stiffness identification method for bridge monitoring. Structural Health Monitoring, 2015. 14(4): p. 14.

27. Gonzalez, A., Covian, E. and Madera, J. Determination of Bridge Natural Frequencies Using a Moving Vehicle Instrumented with Accelerometers and GPS in Ninth International Conference on Computational Structures Technology (CST2008) 2008. Athens, Greece: Civil-Comp Press.

28. Kim, C.W., Isemoto, R., Toshinami, T., Kawatani, M., McGetrick, P.J. \& OBrien, E.J., Experimental investigation of drive-by inspection, in 5th International Conference on Structural Health Monitoring of Intelligent Infrastructure (SHMII-5)2011: Cancun, Mexico.

29. Gonzalez, A., OBrien, E. and McGetrick, P., 'Identification of damping in a bridge using a moving instrumented vehicle. Journal of Sound and Vibration, 2012. 331(18): p. 4115-4131. 
30. Keenahan, J., OBrien, E.J., McGetrick, P.J. and González, A, The use of a dynamic trucktrailer drive-by system to monitor bridge damping. Structural Health Monitoring, 2014. 13(2): p. 183-197.

31. Yang, Y.B., Y.C. Li, and K.C. Chan, Constructing the mode shapes of a bridge from a passing vehicle: a theoretical study. Smart Structures and Systems, 2014. 13(5): p. 22.

32. Oshima, Y., K. Yamamoto, and K. Sugiura, Damage assessment of a bridge based on mode shapes estimated by responses of passing vehicles. Smart Structures and Systems, 2014. 13(5): p. 22.

33. OBrien, E.J., P.J. McGetrick, and A. González, A drive-by inspection system via vehicle moving force identification. Smart Structures and Systems, 2014. 13(5): p. 27.

34. Nguyen, K.V. and H.T. Tran, Multi-cracks detection of a beam-like structure based on the onvehicle vibration signal and wavelet analysis. Journal of Sound and Vibration, 2010. 329(21): p. 4455-4465.

35. Zhang, Y., S.T. Lie, and Z. Xiang, Damage detection method based on operating deflection shape curvature extracted from dynamic response of a passing vehicle. Mechanical Systems and Signal Processing, 2013. 35(1-2): p. 238-254.

36. Malekjafarian, A., P.J. McGetrick, and E.J. OBrien, A Review of Indirect Bridge Monitoring Using Passing Vehicles. Shock and Vibration, 2015. 2015: p. 16.

37. Tedesco, J.W., W.G. Mcdougal, and C.A. Ross, Structural dynamics: theory and applications. 1999, California, USA: Addison Wesley Longman, Inc.

38. Kwon, Y.W. and H. Bang, The finite element method using MATLAB. 2000: CRC Press, Inc., Boca Raton, FL.

39. Mahmoud, M.A., Effect of cracks on the dynamic response of a simple beam subject to a moving load. Proceedings of the Institution of Mechanical Engineers, Part F: Journal of Rail and Rapid Transit, 2001(3): p. 207-215.

40. Hou, Z., M.N. Noori, and R. St Amand, Wavelet-based approach for structural damage detection. Journal of Engineering Mechanics, 2000. 126(7).

41. Daubechies, I., Ten lectures on wavelets. 2006: Society for industrial and applied mathematics.

42. Gentile, A. and A. Messina, On the continuous wavelet transforms applied to discrete vibrational data for detecting open cracks in damaged beams. International Journal of Solids and Structures, 2003. 40: p. 295-31.

43. Mallat, S.G., A wavelet tour of signal processing. 1999, London, UK: Academic Press.

44. Reda Taha, M.M., et al., Wavelet transform for structural health monitoring: a compendium of uses and features. Structural Health Monitoring, 2006. 5(3): p. 267-295.

45. Gonzalez, A. and D. Hester, An Investigation Into The Acceleration Response of a Damaged Beam-Type Structure to a Moving Load. Journal of Sound and Vibration, 2013. 332: p. 16.

46. Cantero, D., A. González, and E.J. O'Brien, Comparison of bridge dynamic amplification due to articulated 5-axle trucks and large cranes. Baltic Journal of Road and Bridge Engineering, 2011. 6(1): p. 39-47.

47. El-Madany, M.M., Design and optimization of truck suspensions using covariance analysis. Computers \& structures, 1988. 28(2): p. 241-246.

48. Cebon, D., Handbook of vehicle-road interaction. 1999, Netherlands: Swets \& Zeitlinger.

49. Chang, K.C., C.W. Kim, and S. Borjigin, Variability in bridge frequency induced by a parked vehicle. Smart Structures and Systems, 2014. 13(5): p. 18.

50. Gonzalez, A., et al., Determination of bridge lifetime dynamic amplification factor using finite element analysis of critical loading scenarios. Engineering structures, 2008. 30: p. 7.

51. Yang, Y.B., J.D. Yau, and Y.S. Wu, Vehicle-bridge interaction dynamics: with applications to high-speed railways. 2004: World Scientific Pub Co Inc.

52. González, A., Vehicle-bridge dynamic interaction using finite element modelling, in Finite Element Analysis, D. Moratal, Editor. 2010, Sciyo: Croatia.

53. Yang, F. and G.A. Fonder, An iterative solution method for dynamic response of bridgevehicles systems. Earthquake engineering \& structural dynamics, 1996. 25(2): p. 195-215. 
54. Green, M.F. and D. Cebon, Dynamic interaction between heavy vehicles and highway bridges. Computers \& structures, 1997. 62(2): p. 253-264.

55. Zhu, X.Q. and S.S. Law, DYNAMIC LOAD ON CONTINUOUS MULTI-LANE BRIDGE DECK FROM MOVING VEHICLES. Journal of Sound and Vibration, 2002. 251(4): p. 697-716.

56. Green, M., D. Cebon, and D. Cole, Effects of Vehicle Suspension Design on Dynamics of Highway Bridges. Journal of Structural Engineering, 1995. 121(2): p. 272-282.

57. Standardisation, I.O.f., Mechnical vibration-road surface profiles - reporting of Measure data (ISO 8608 (BS7853:1996)), 1995.

58. Lyons, R., Understanding Digital Signal Processing. Third ed. 2011, Boston, USA: Prentice Hall. 


\section{$970 \quad$ Figures}

971

972

973

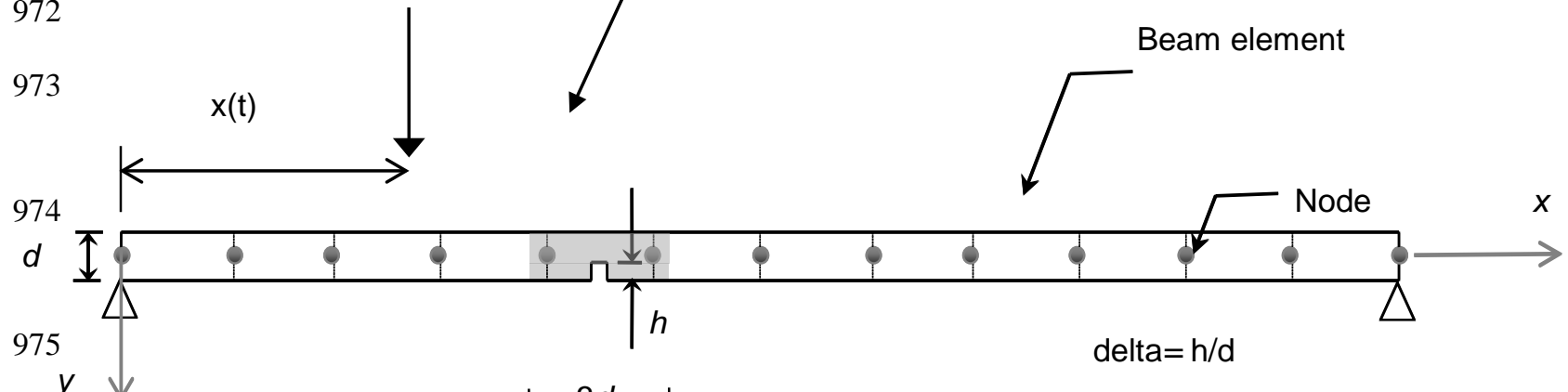

976

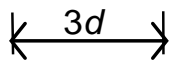

$L$ loss in stiffness

977 Fig. 1, Sketch of discretised beam model subject to a moving force. (Note, $x(t)$ represents the $\mathrm{x}-$ 978 coordinate of the load at time ' $t$ ').

979

980 

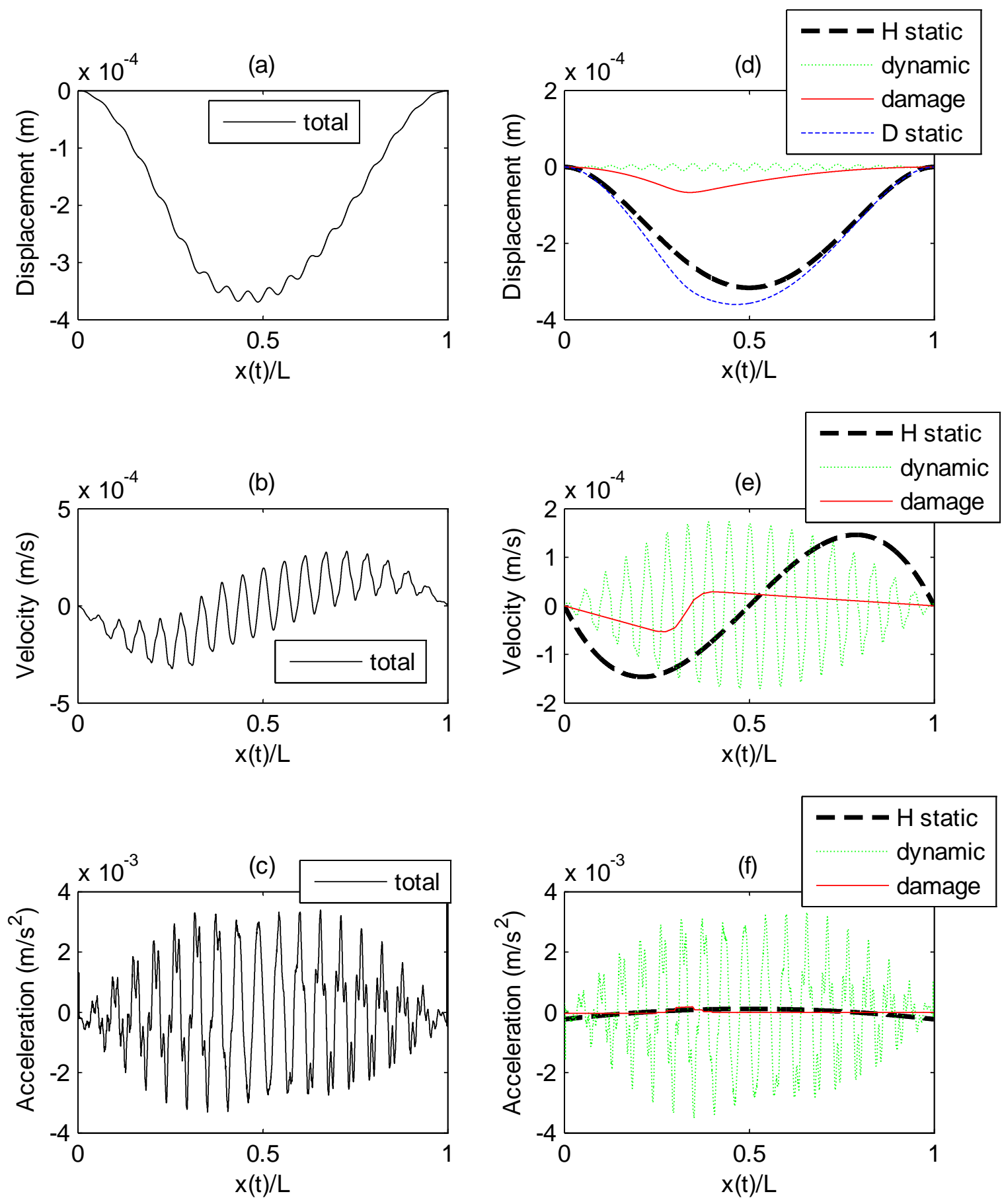

Fig. 2. Total response of the section of bridge deck immediately under the point load and the components of the total response to the force travelling at $6 \mathrm{~m} \mathrm{~s}^{-1}$ : (a) total displacement; (b) total velocity; (c) total acceleration; (d) components of displacement; (e) components of velocity; (f) components of acceleration. 


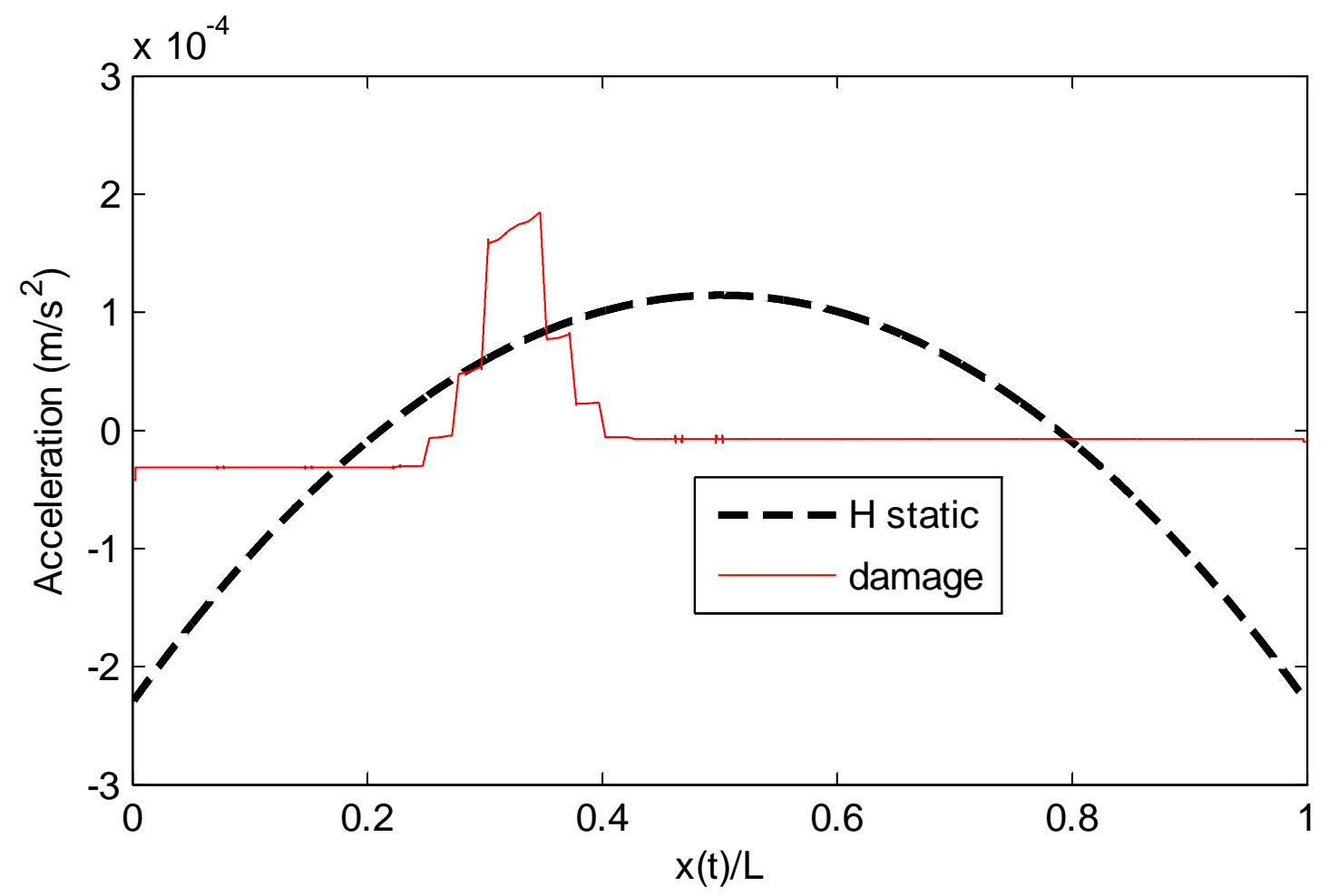

989

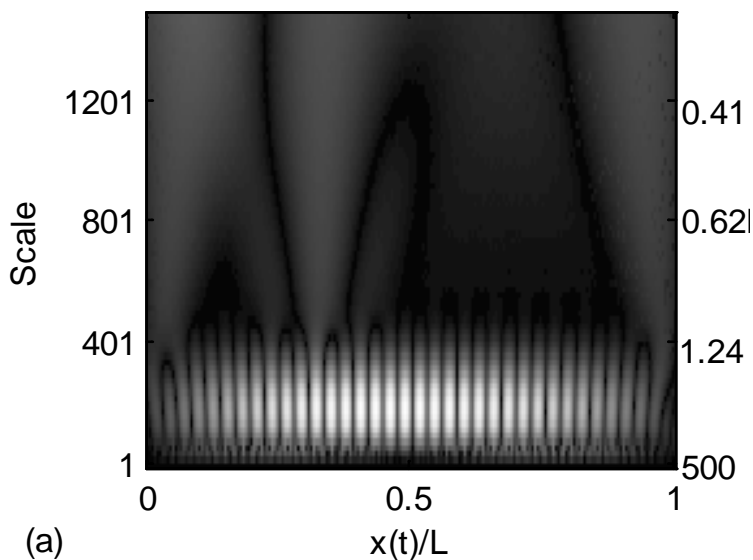

Fig. 3, Magnified view of 'H static' and 'damaged' components (from Fig. 2(f)) of the acceleration response.

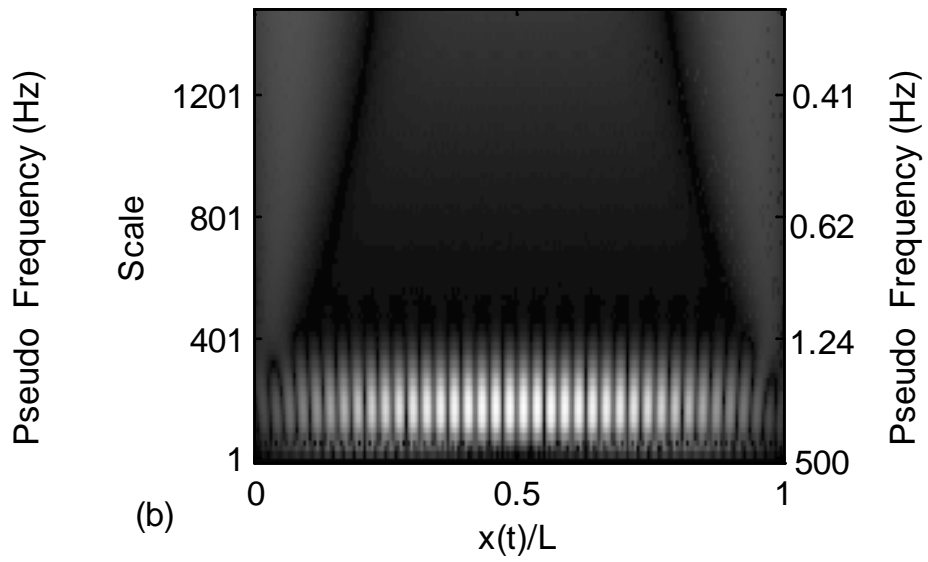

Fig. 4, Applying Wavelet transform to the acceleration signal under a point load travelling at $6 \mathrm{~m} \mathrm{~s}^{-}$ 1: (a) delta $=0.3$ at $0.33 \mathrm{~L}$, (b) delta $=0.0$. 

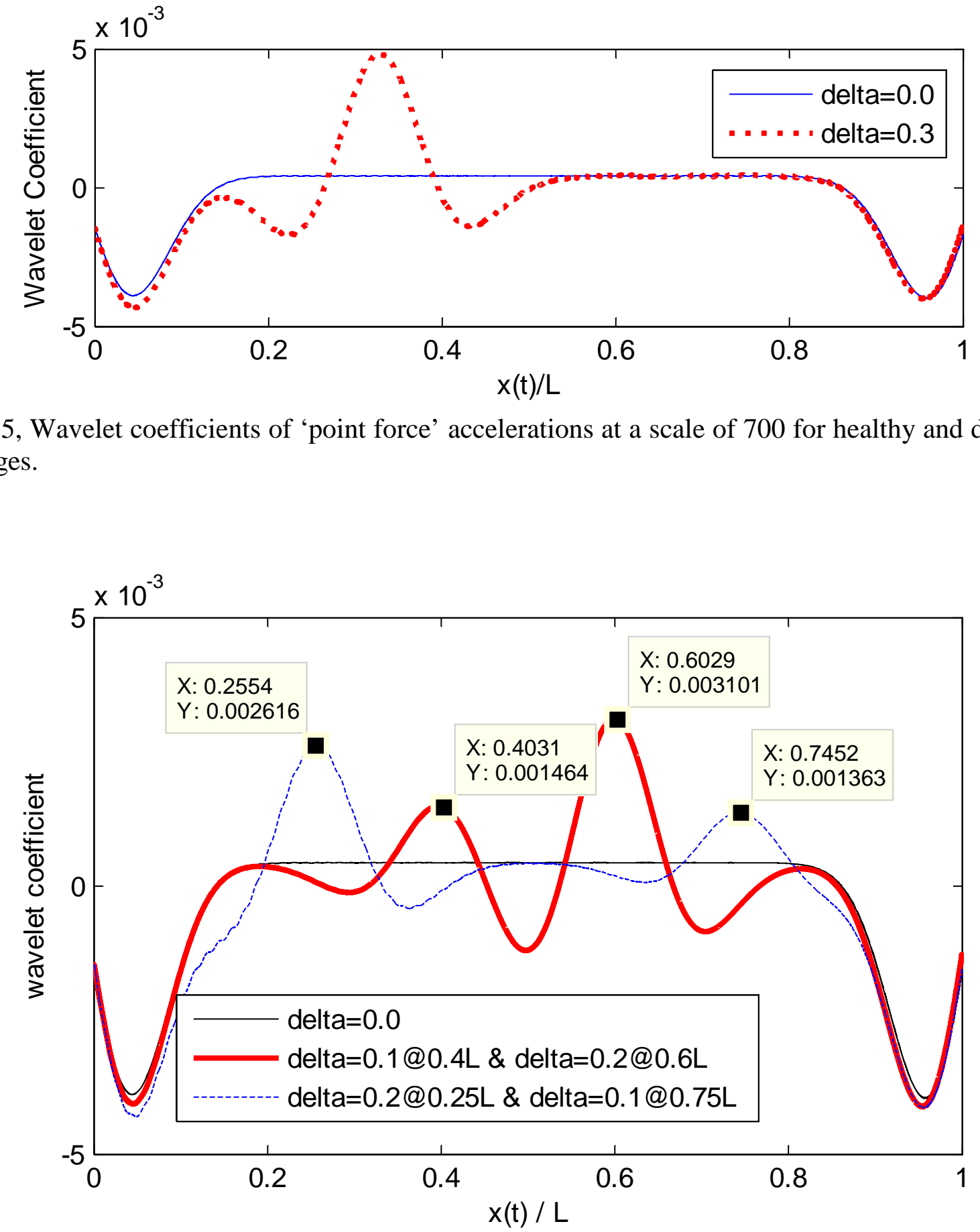

Fig. 6, Wavelet coefficients of 'point force' accelerations at a scale of 700 for healthy bridge (delta $=0.0$ ) and two cases where the bridge has more than one damage location.

Fig. 5, Wavelet coefficients of 'point force' accelerations at a scale of 700 for healthy and damaged bridges. 
(a) mexh

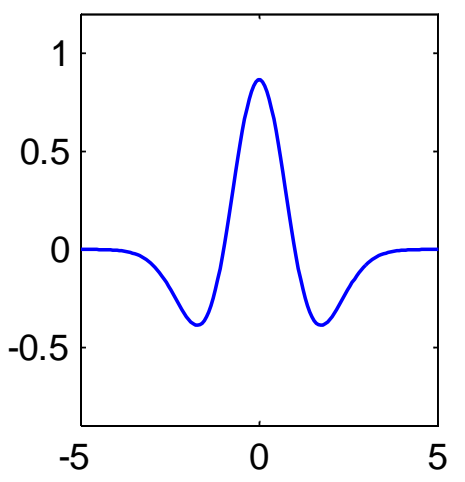

(b) gaus2

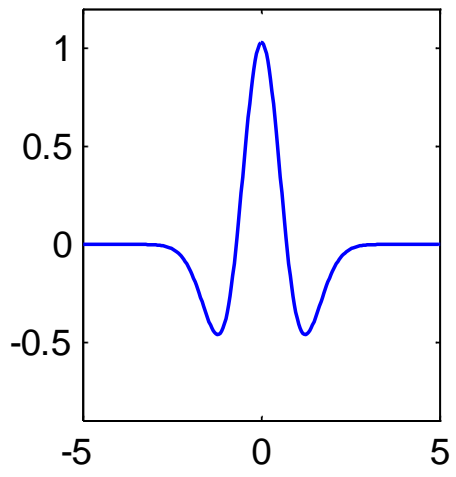

(c) gaus8

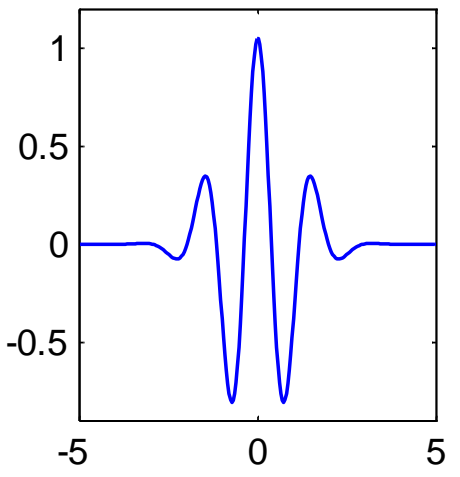

1016

1018

1019

1020

1021

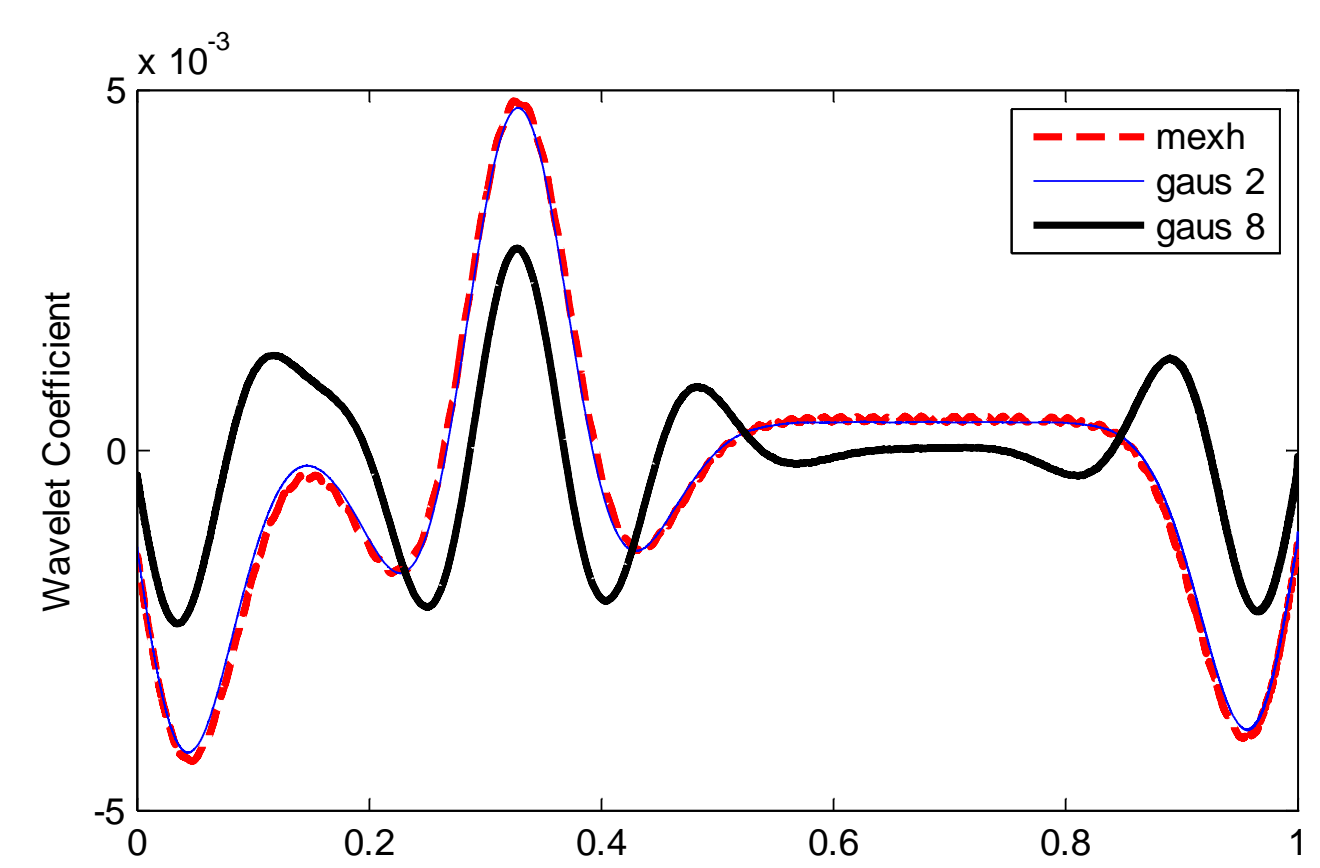

1022

1023

1024

1025

1026

1027

1028

Fig. 7, Different wavelets (a) Mexican Hat, (b) Gauss 2, (c) Gauss 8.

Fig. 8, results of analyzing the signal in Fig. 2(c) with the wavelets shown in Fig. 7. 

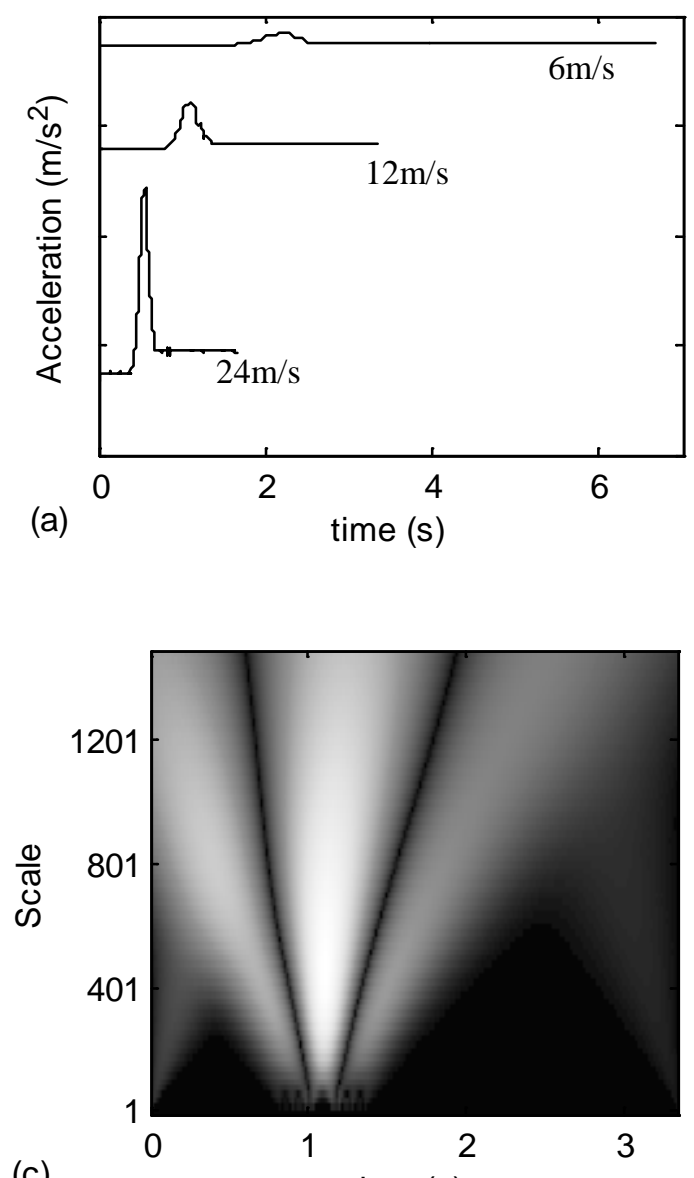

(c) time (s)
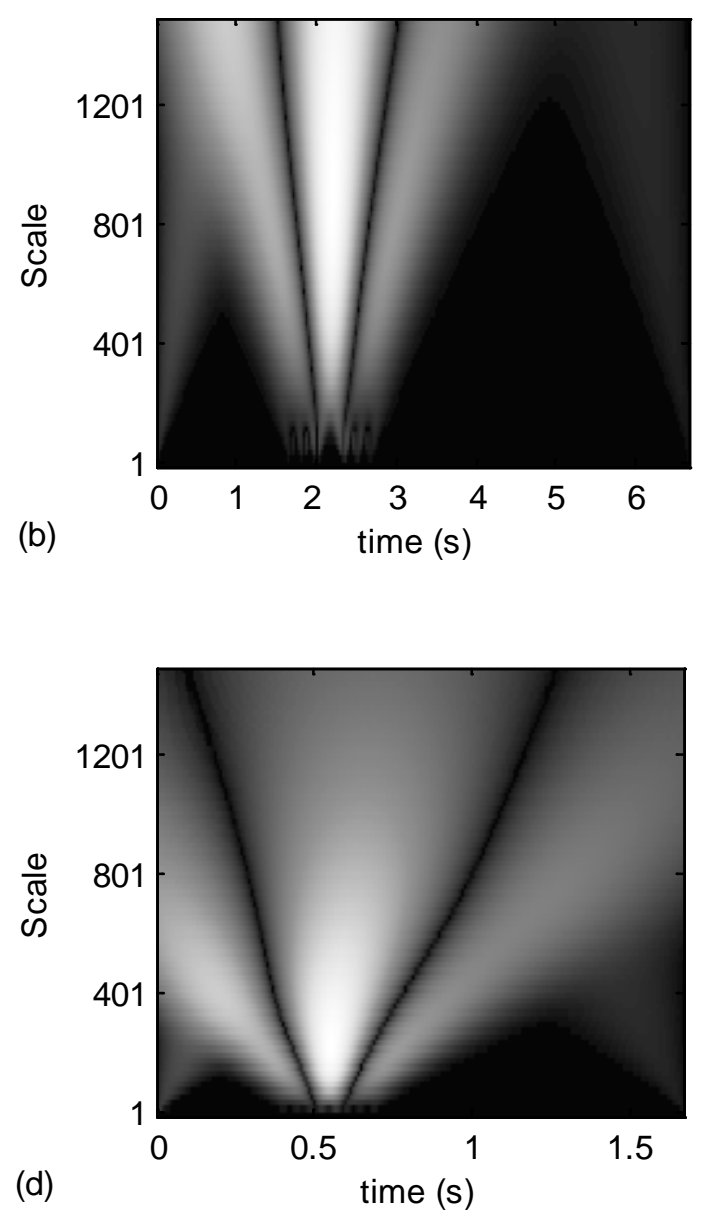

Fig. 9, Effect of load speed on the scales at which 'damage' component can be detected, : (a) 'damage' components for speeds of 6,12 and $24 \mathrm{~ms}^{-1}$ plotted in the time domain, (b) wavelet transform of the $6 \mathrm{~ms}^{-1}$ 'damage' component shown in (a), (c) wavelet transform of the $12 \mathrm{~ms}^{-1}$ damage component shown in (a), (d) wavelet transform of the $24 \mathrm{~ms}^{-1}$ damage component shown in (a).

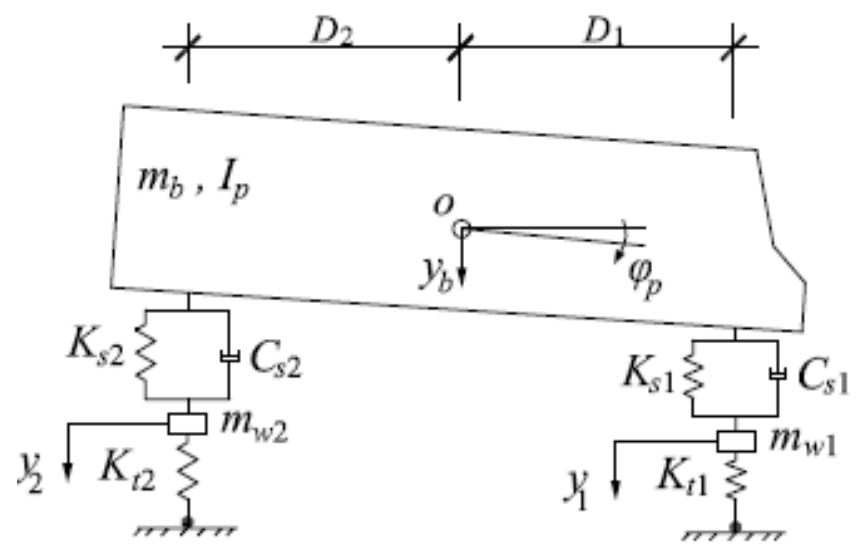

Fig. 10, Schematic of the vehicle model 

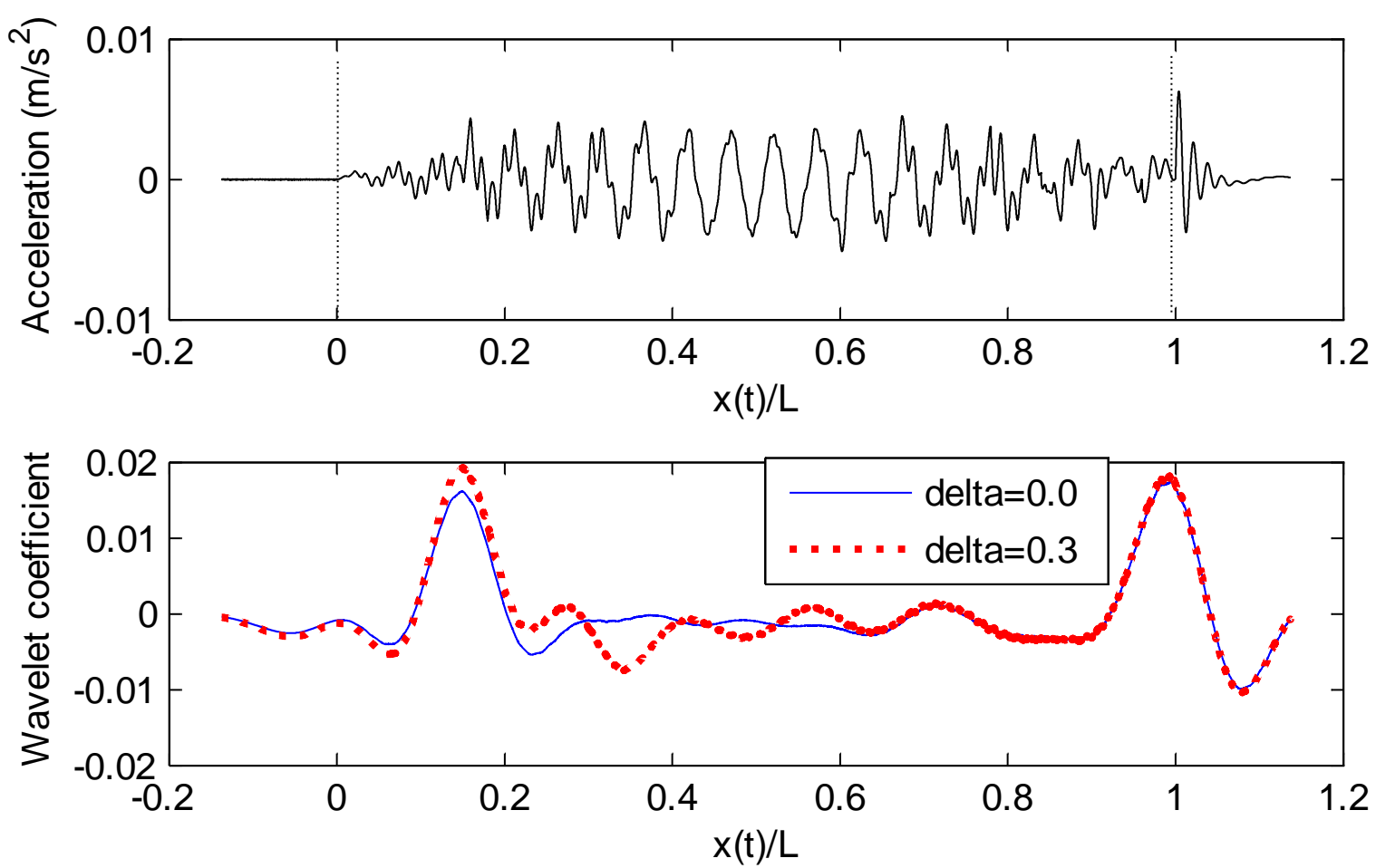

Fig. 11, Analysis of axle acceleration due to a half-car vehicle model travelling over a bridge with a smooth profile at $6 \mathrm{~ms}^{-1}$ : (a) axle 1 acceleration signal, (b) wavelet transform of healthy and damaged acceleration signals at a scale of 700 using Mexican hat wavelet.

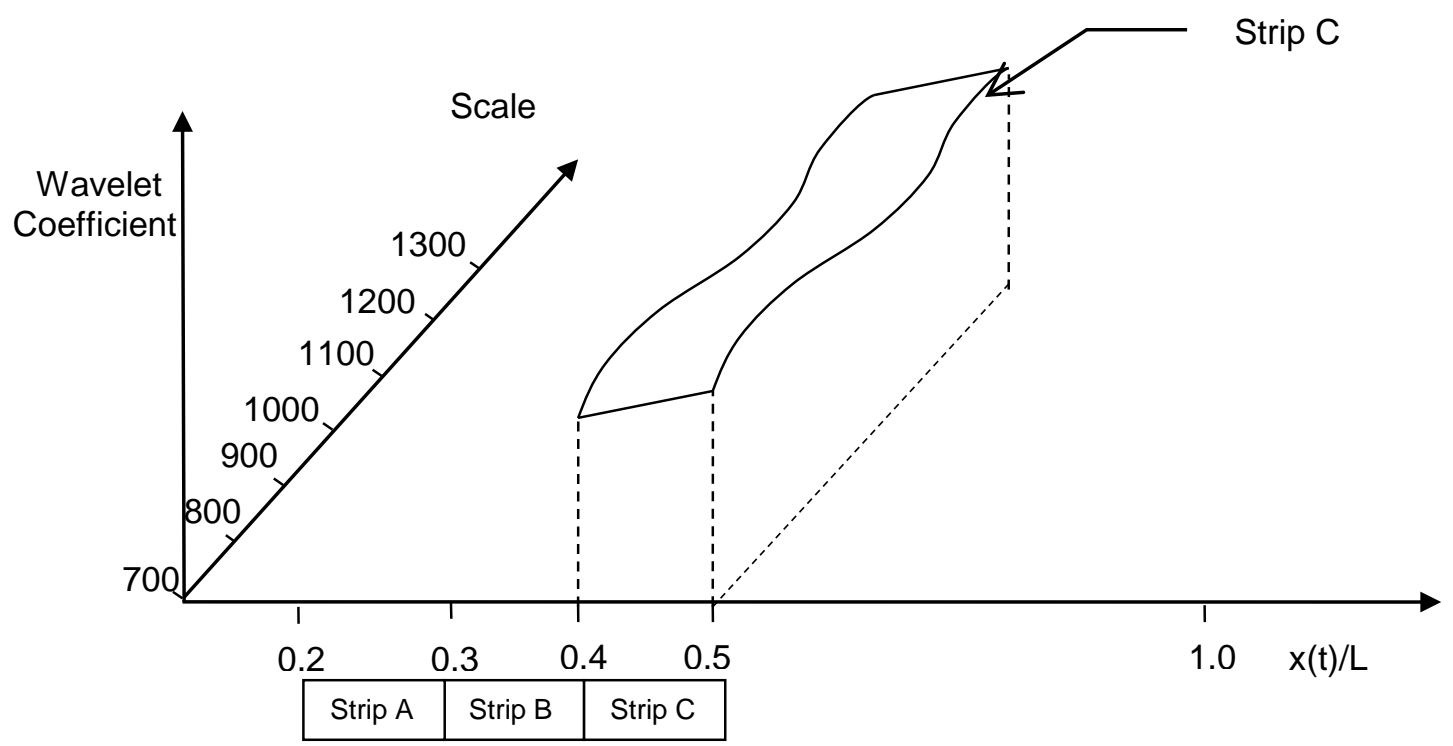

Fig. 12, Sketch showing strip C of a theoretical 3D wavelet surface. 


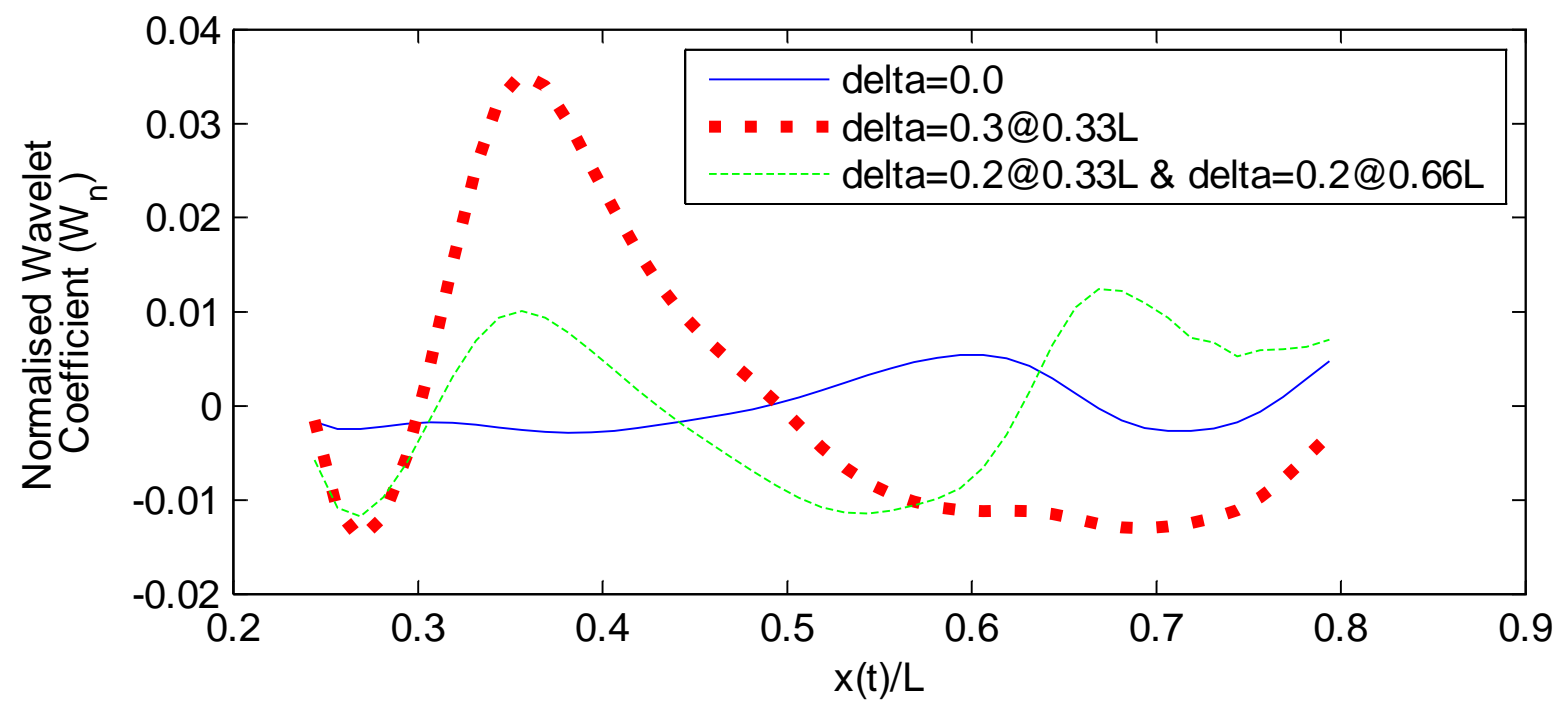

Fig. 13, Normalised wavelet coefficient for axle accelerations over healthy and damaged bridges

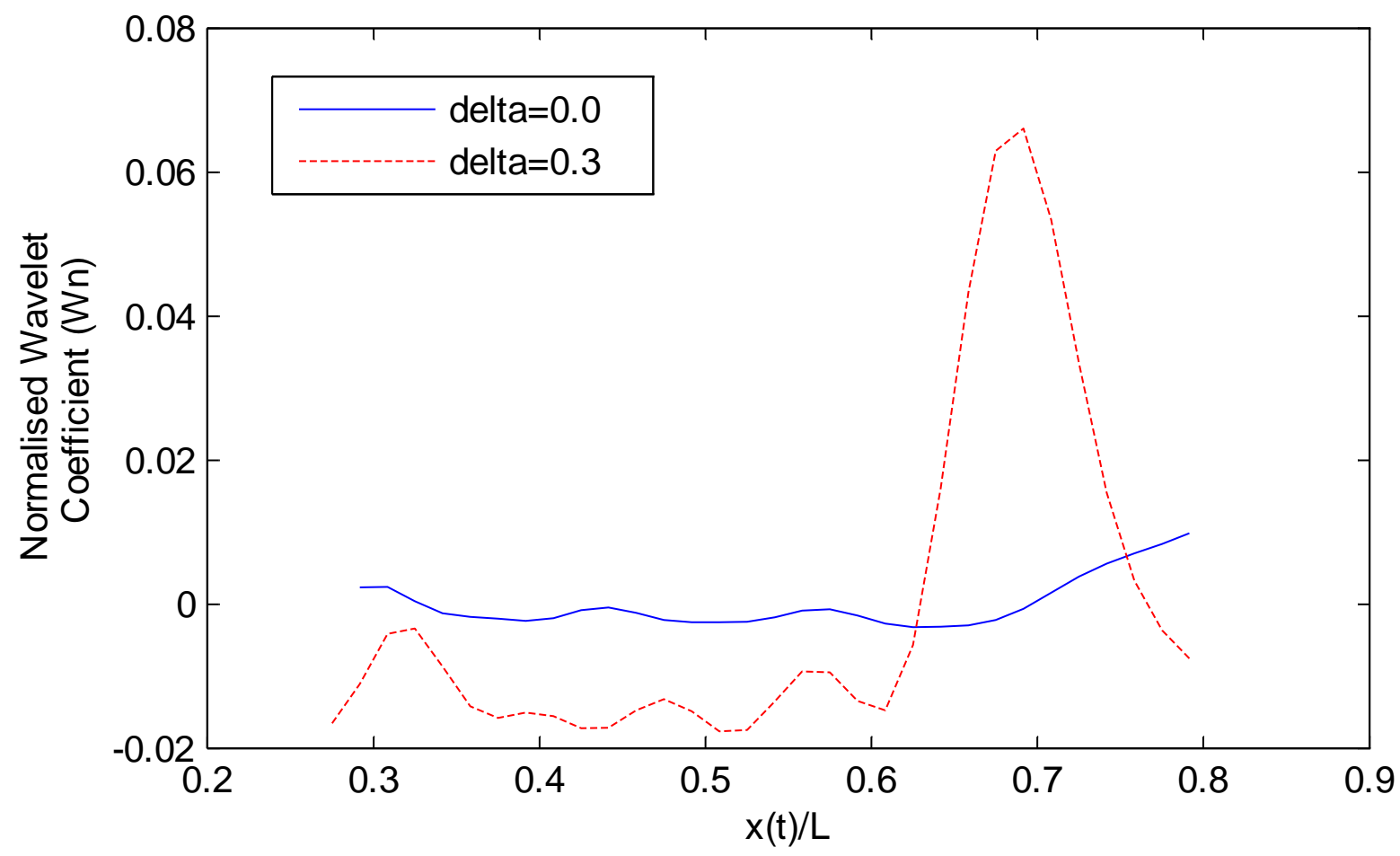

Fig. 14, Normalised wavelet coefficient for axle accelerations over healthy and damaged bridge. (Bridge span $30 \mathrm{~m}$ ) 


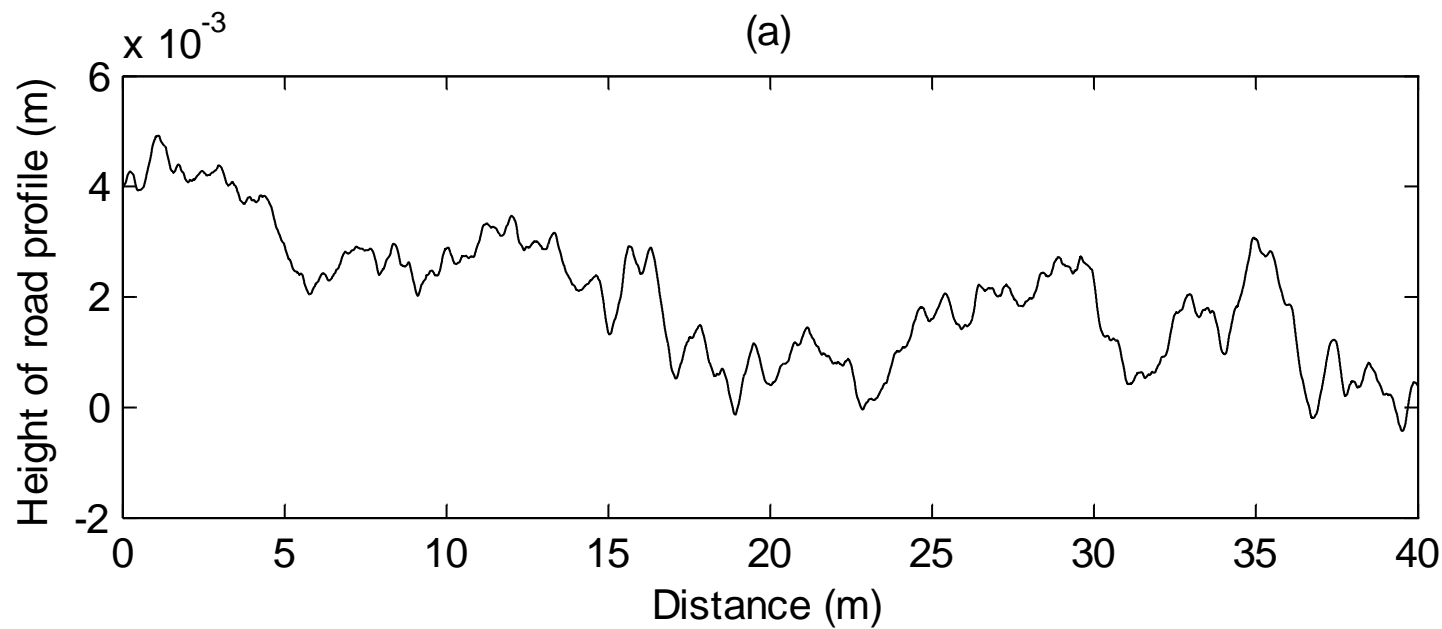

(b)

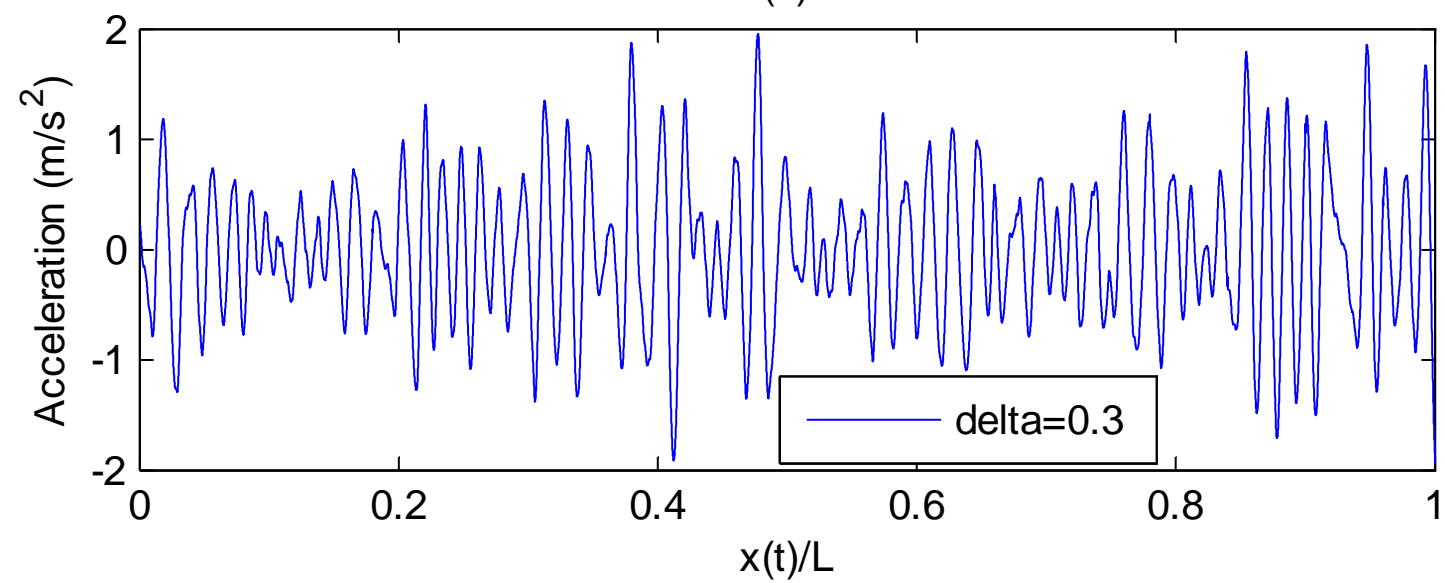

Fig. 15, Simulated road profile and acceleration signal from axle 1: (a) class A road profile on the bridge, (b) axle 1 acceleration when driving over road profile defined in (a) at $6 \mathrm{~ms}^{-1}$.

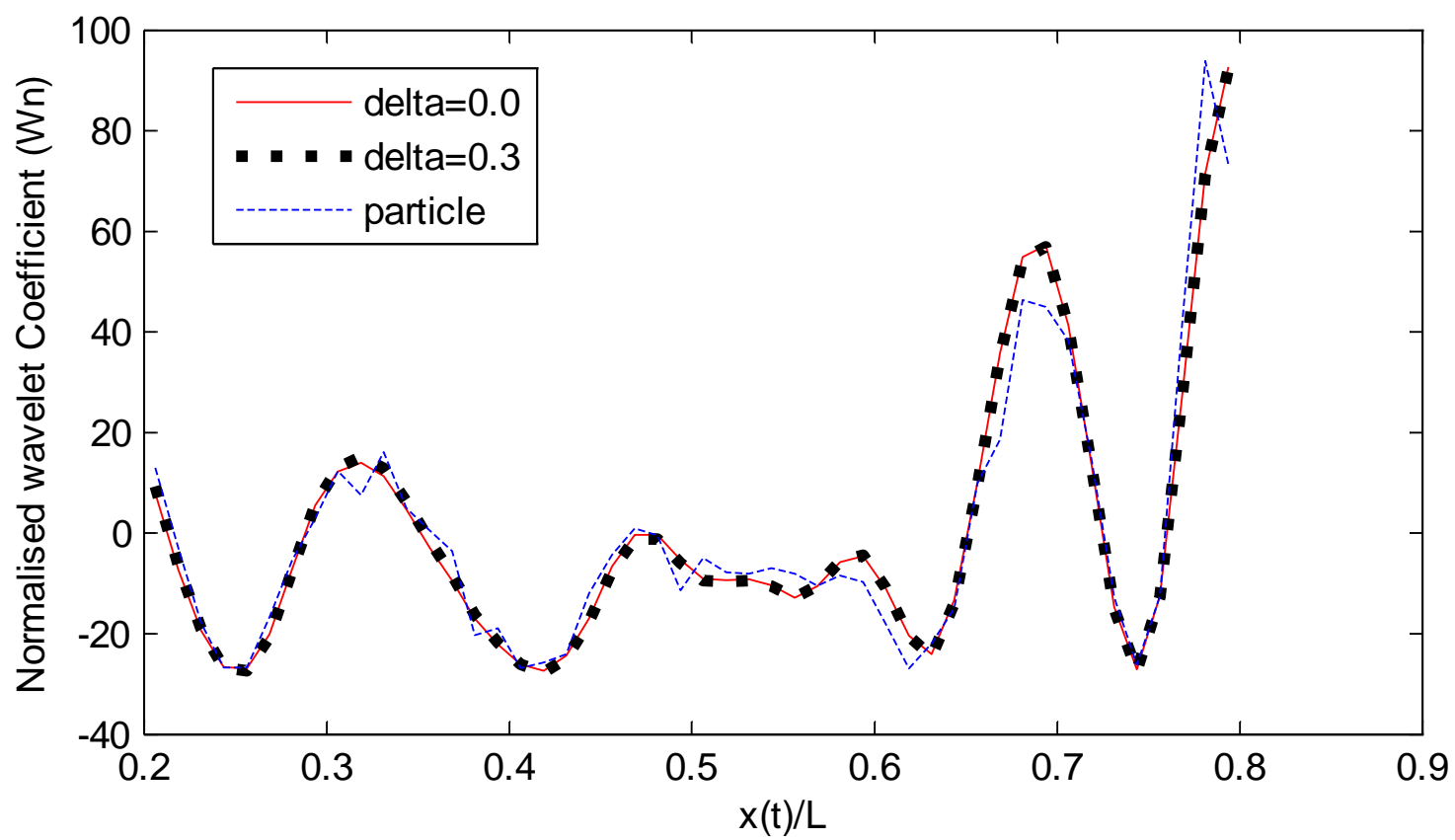

Fig. 16, Normalized wavelet coefficient calculated from axle acceleration signals relating to the healthy bridge (delta $=0.0$ ), the damaged bridge (delta $=0.3$ ), and a theoretical particle traversing the 'solid' road profile. 


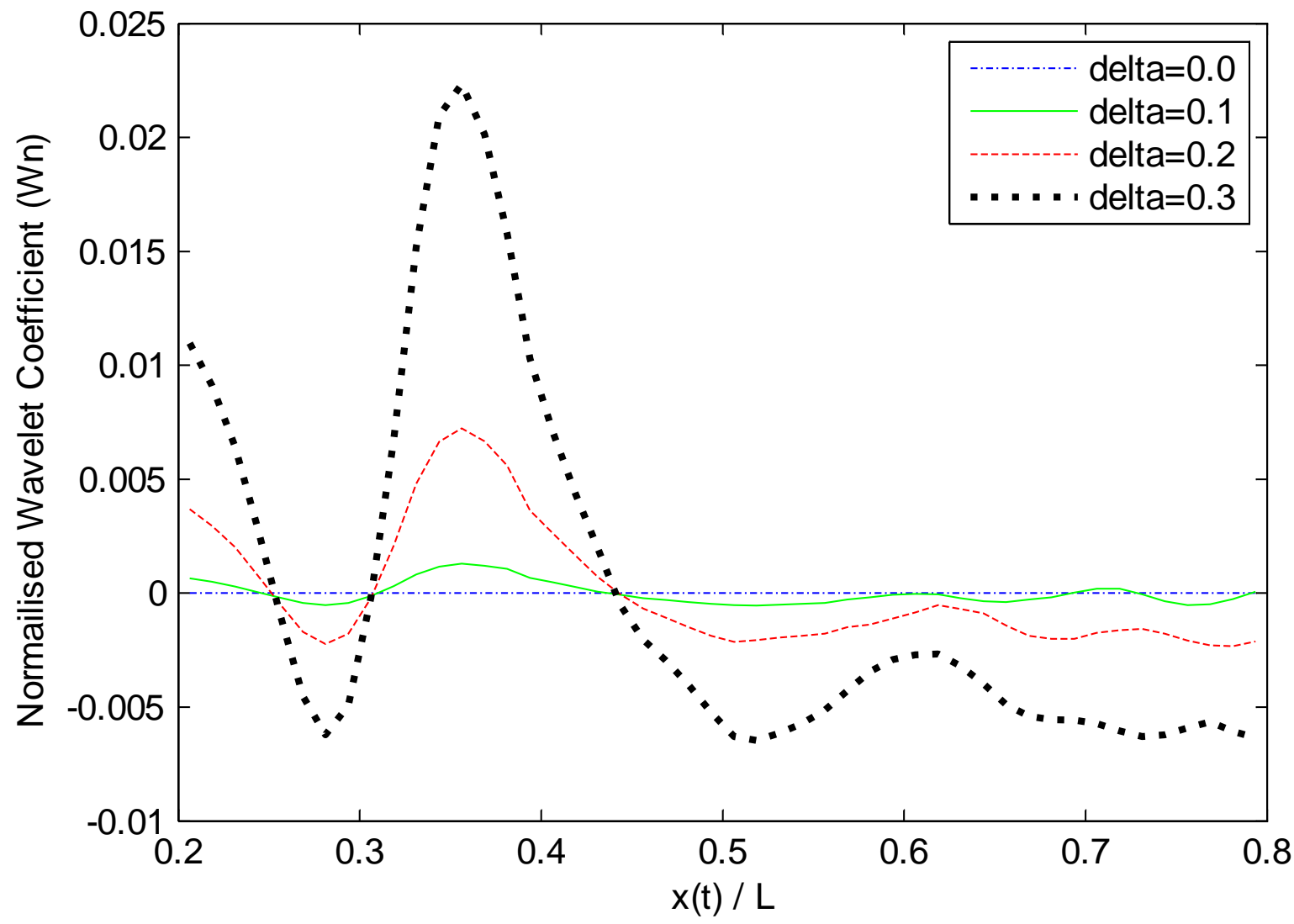

1073

1074 Fig. 17, Normalized wavelet coefficients calculated from the residual wavelet surfaces 1075 corresponding to damage at the $1 / 3$ point of the span with severities of delta $=0.0$ to delta $=0.3$. 
(a)

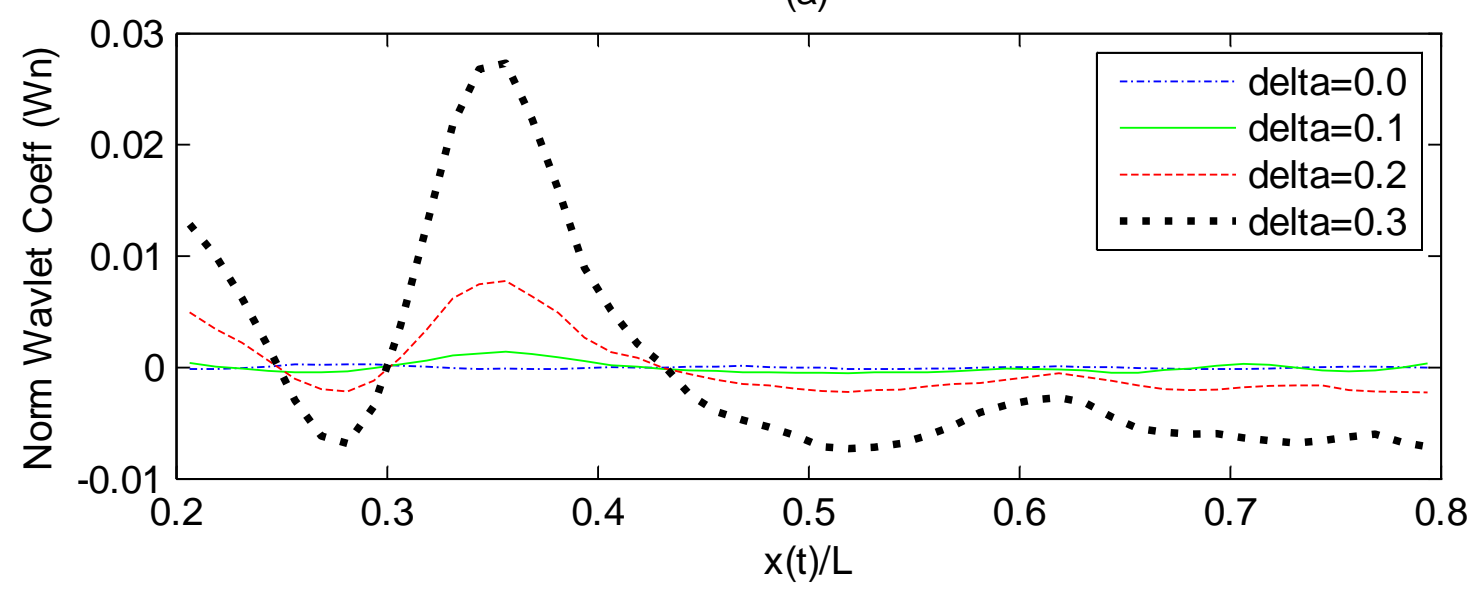

(b)

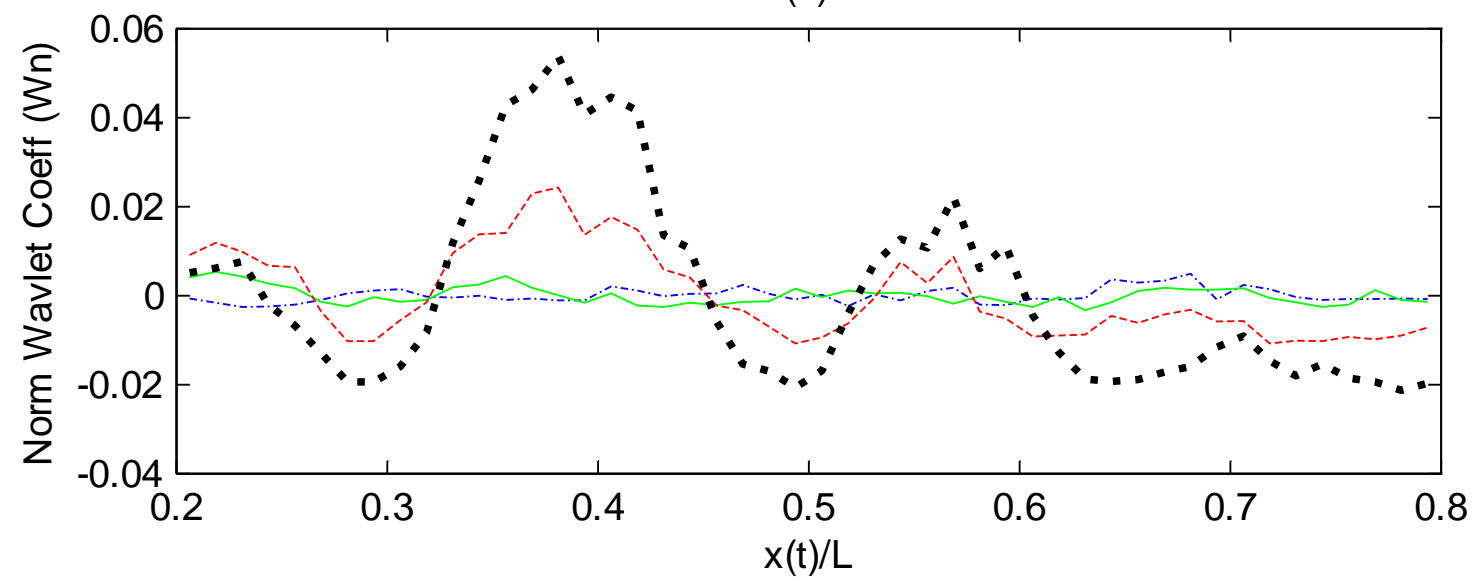

(c)

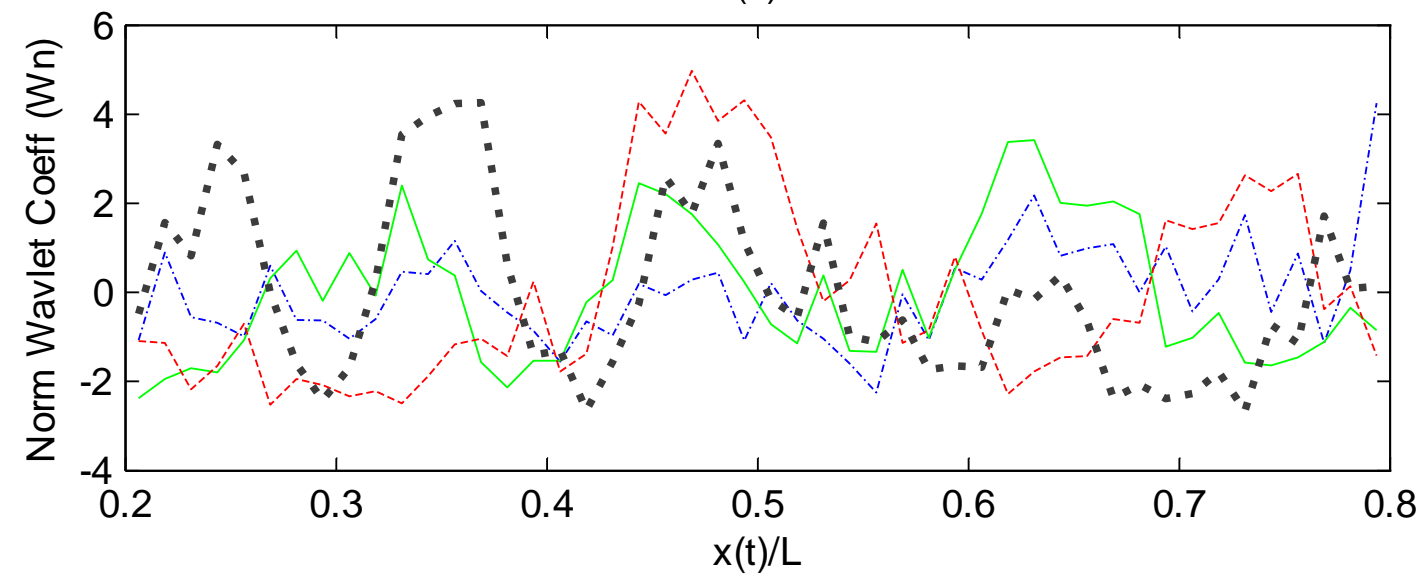

Fig. 18, Normalized wavelet coefficients calculated from noise corrupted wavelet surfaces when there is damage at the $1 / 3$ point of the span with severities of delta $=0.0$ to delta $=0.3$ : (a) Signal to Noise Ratio of 70, (b) Signal to Noise Ratio of 50, (c) Signal to Noise Ratio of 20. 


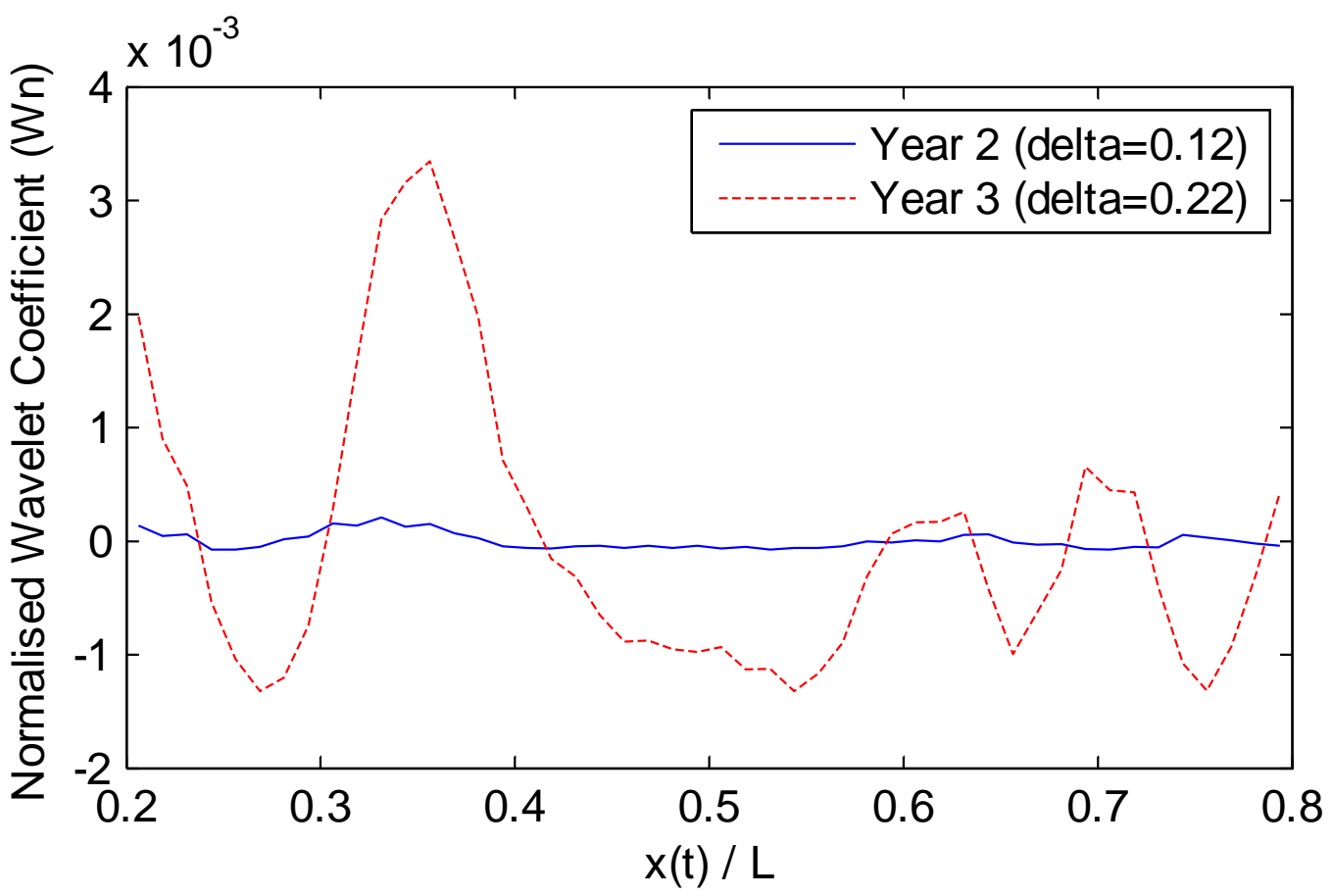

1082

1083 1084 1085 1086 1087 1088

Fig. 19, Normalized wavelet coefficients calculated from residual wavelet surfaces based on a datum from a bridge with delta $=0.1(\mathrm{SNR}=70)$ 